\title{
SOME ESTIMATES OF SCHRÖDINGER TYPE OPERATORS ON VARIABLE LEBESGUE AND HARDY SPACES
}

\author{
JUNQIANG ZHANG ${ }^{1 *}$ and ZONGGUANG LIU $^{2}$
}

\begin{abstract}
In this article, the authors consider the Schrödinger type operator $L:=-\operatorname{div}(A \nabla)+V$ on $\mathbb{R}^{n}$ with $n \geq 3$, where the matrix $A$ satisfies uniformly elliptic condition and the nonnegative potential $V$ belongs to the reverse Hölder class $R H_{q}\left(\mathbb{R}^{n}\right)$ with $q \in(n / 2, \infty)$. Let $p(\cdot): \mathbb{R}^{n} \rightarrow(0, \infty)$ be a variable exponent function satisfying the globally log-Hölder continuous condition. When $p(\cdot): \mathbb{R}^{n} \rightarrow(1, \infty)$, the authors prove that the operators $V L^{-1}, V^{1 / 2} \nabla L^{-1}$ and $\nabla^{2} L^{-1}$ are bounded on variable Lebesgue space $L^{p(\cdot)}\left(\mathbb{R}^{n}\right)$. When $p(\cdot): \mathbb{R}^{n} \rightarrow(0,1]$, the authors introduce the variable Hardy space $H_{L}^{p(\cdot)}\left(\mathbb{R}^{n}\right)$, associated to $L$, and show that $V L^{-1}, V^{1 / 2} \nabla L^{-1}$ and $\nabla^{2} L^{-1}$ are bounded from $H_{L}^{p(\cdot)}\left(\mathbb{R}^{n}\right)$ to $L^{p(\cdot)}\left(\mathbb{R}^{n}\right)$.
\end{abstract}

\section{INTRODUCTION AND MAIN RESULTS}

Let $n \geq 3$ and consider the Schrödinger operator $-\Delta+V$ on the Euclidean space $\mathbb{R}^{n}$, where $\Delta:=\sum_{j=1}^{n} \frac{\partial^{2}}{\partial x_{j}^{2}}$ denotes the Laplacian operator on $\mathbb{R}^{n}$ and $V$ is a nonnegative potential. If $V$ is a nonnegative polynomial on $\mathbb{R}^{n}$, Zhong [29] showed that the operators $\nabla(-\Delta+V)^{-1 / 2}, \nabla^{2}(-\Delta+V)^{-1}$ and $\nabla(-\Delta+V)^{-1} \nabla$ are the classical Calderón-Zygmund operators which are bounded on $L^{p}\left(\mathbb{R}^{n}\right)$ for any $p \in(1, \infty)$.

Then, in 1995, Shen [21] proved that if $V$ belongs to the reverse Hölder class $R H_{q}\left(\mathbb{R}^{n}\right)$ with $q \in[n, \infty)$, denoted by $V \in R H_{q}\left(\mathbb{R}^{n}\right)$, then $\nabla(-\Delta+V)^{-1 / 2}$, $(-\Delta+V)^{-1 / 2} \nabla$ and $\nabla(-\Delta+V)^{-1} \nabla$ are Calderón-Zygmund operators. Recall that a nonnegative measurable function $V$ on $\mathbb{R}^{n}$ is said to belong to the reverse Hölder class $R H_{q}\left(\mathbb{R}^{n}\right), q \in[1, \infty]$, if $V \in L_{\text {loc }}^{q}\left(\mathbb{R}^{n}\right)$ and there exists a positive constant $C$ such that, for any ball $B \subset \mathbb{R}^{n}$,

$$
\left\{\frac{1}{|B|} \int_{B}[V(x)]^{q} d x\right\}^{1 / q} \leq C \frac{1}{|B|} \int_{B} V(x) d x,
$$

where we replace $\left\{\frac{1}{|B|} \int_{B}[V(x)]^{q} d x\right\}^{1 / q}$ by $\|V\|_{L^{\infty}(B)}$ when $q=\infty$. If $V \in$ $R H_{q}\left(\mathbb{R}^{n}\right)$ with $q \in[n / 2, \infty)$, Shen [21] also obtained the $L^{p}\left(\mathbb{R}^{n}\right)$-boundedness of $V(-\Delta+V)^{-1}, V^{1 / 2} \nabla(-\Delta+V)^{-1}$ and $\nabla^{2}(-\Delta+V)^{-1}$ for any $p \in\left(1, p_{0}\right)$, where $p_{0} \in(1, \infty)$ is a constant which may depend on $n$ and $q$. Noticing that if $V$ is a nonnegative polynomial on $\mathbb{R}^{n}$, then $V \in R H_{\infty}\left(\mathbb{R}^{n}\right) \subset R H_{q}\left(\mathbb{R}^{n}\right)$ for

Copyright 2018 by the Tusi Mathematical Research Group.

Date: Received: xxxxxx; Revised: yyyyyy; Accepted: zzzzzz.

*Corresponding author.

2010 Mathematics Subject Classification. Primary 42B20; Secondary 35J10, 42B35.

Key words and phrases. Schrödinger type operator, reverse Hölder class, variable Lebesgue space, variable Hardy spaces, and atom. 
any $q \in[1, \infty)$ (see [21, p. 516]), hence, Shen [21] generalized the results in [29]. Moreover, for the weighted $L^{p}\left(\mathbb{R}^{n}\right)$ boundedness of these operators, we refer the reader to $[17,23]$.

For $p \in(0,1]$, it is well-known that many classical operators are bounded on Hardy spaces $H^{p}\left(\mathbb{R}^{n}\right)$, but not on $L^{p}\left(\mathbb{R}^{n}\right)$, for example, the Riesz transforms $\nabla(-\Delta)^{-1 / 2}$ and $\nabla^{2}(-\Delta)^{-1}$ (see [22]). However, when working with some differential operators other than the Laplacian operator, the classical Hardy spaces $H^{p}\left(\mathbb{R}^{n}\right)$ are not suitable any more, since $H^{p}\left(\mathbb{R}^{n}\right)$ is intimately connected with the Laplacian operator. This motivates people to develop a theory of Hardy spaces $H_{L}^{p}\left(\mathbb{R}^{n}\right)$ associated with different operators $L$. This topic has attracted a lot of attention in the last decades, which can be found in [1, 10, 13, 24]. In particular, it is showed in $[12,14]$ that the Riesz transform $\nabla(-\Delta+V)^{-1 / 2}$ is bounded from the Hardy space $H_{-\Delta+V}^{p}\left(\mathbb{R}^{n}\right)$ to $L^{p}\left(\mathbb{R}^{n}\right)$ for $p \in(0,1]$ and bounded on $H^{p}\left(\mathbb{R}^{n}\right)$ for $p \in\left(\frac{n}{n+1}, 1\right]$, where $V$ is a nonnegative potential on $\mathbb{R}^{n}$. Moreover, as a generalization of the results in [21] for $p \leq 1, \mathrm{~F}$. K. Ly [16] proved that, for any $p \in(0,1]$, the operators $\nabla^{2}(-\Delta+V)^{-1}$ and $V(-\Delta+V)^{-1}$ are bounded from $H_{-\Delta+V}^{p}\left(\mathbb{R}^{n}\right)$ to $L^{p}\left(\mathbb{R}^{n}\right)$ and, for any $p \in\left(\frac{n}{n+1}, 1\right], \nabla^{2}(-\Delta+V)^{-1}$ is bounded from $H_{-\Delta+V}^{p}\left(\mathbb{R}^{n}\right)$ to $H^{p}\left(\mathbb{R}^{n}\right)$, where $V \in R H_{q}\left(\mathbb{R}^{n}\right)$ with $q>\max \{n / 2,2\}$. Moreover, Cao et al. [4] introduced Musielak-Orlicz-Hardy space $H_{\varphi,-\Delta+V}\left(\mathbb{R}^{n}\right)$ and, via establishing its atomic decomposition, they obtained the boundedness of $V(-\Delta+V)^{-1}$ and $\nabla^{2}(-\Delta+V)^{-1}$ on $H_{\varphi,-\Delta+V}\left(\mathbb{R}^{n}\right)$, where $\varphi: \mathbb{R}^{n} \times[0, \infty) \rightarrow[0, \infty)$ is a Musielak-Orlicz function. Observe that the Musielka-Orlicz-Hardy space is a more generalized space which unifies the Hardy space, the weighted Hardy space, the Orlicz-Hardy space and the weighted Orlicz-Hardy space.

In this paper, we consider the Schrödinger type operator

$$
L:=-\operatorname{div}(A \nabla)+V \text { on } \mathbb{R}^{n}, n \geq 3,
$$

where $V$ is a nonnegative potential and $A:=\left\{a_{i j}\right\}_{1 \leq i, j \leq n}$ is a matrix of measurable functions satisfying the following conditions:

Assumption 1.1. There exists a constant $\lambda \in(0,1]$ such that, for any $x, \xi \in \mathbb{R}^{n}$,

$$
a_{i j}(x)=a_{j i}(x) \quad \text { and } \quad \lambda|\xi|^{2} \leq \sum_{i, j=1}^{n} a_{i j}(x) \xi_{i} \xi_{j} \leq \lambda^{-1}|\xi|^{2} .
$$

Assumption 1.2. There exist constants $\alpha \in(0,1]$ and $K \in(0, \infty)$ such that, for any $i, j \in\{1, \ldots, n\}$,

$$
\left\|a_{i j}\right\|_{C^{\alpha}\left(\mathbb{R}^{n}\right)} \leq K
$$

where $C^{\alpha}\left(\mathbb{R}^{n}\right)$ denotes the set of all functions $f$ satisfying the $\alpha$-Hölder contition

$$
\|f\|_{C^{\alpha}\left(\mathbb{R}^{n}\right)}:=\sup _{x, y \in \mathbb{R}^{n}, x \neq y} \frac{|f(x)-f(y)|}{|x-y|^{\alpha}}<\infty .
$$

Assumption 1.3. There exists a constant $\alpha \in(0,1]$ such that, for any $i, j \in$ $\{1, \ldots, n\}, x \in \mathbb{R}^{n}$ and $z \in \mathbb{Z}^{n}$,

$$
a_{i j} \in C^{1+\alpha}\left(\mathbb{R}^{n}\right), \quad a_{i j}(x+z)=a_{i j}(x) \quad \text { and } \quad \sum_{k=1}^{n} \partial_{k}\left(a_{i j}(\cdot)\right)(x)=0 .
$$


For the Schrödinger type operator $L=-\operatorname{div}(A \nabla)+V$, under the assumption that $V \in R H_{\infty}\left(\mathbb{R}^{n}\right)$ and $A$ satisfies some of Assumptions 1.1, 1.2 and 1.3 below, Kurata and Sugano [15] established the boundedness of $V L^{-1}, V^{1 / 2} \nabla L^{-1}$ and $\nabla^{2} L^{-1}$ on the weighted Lebesgue spaces and Morrey spaces. Recently, motivated by [4] and [15], Yang [25] considered the boundedness of $V L^{-1}, V^{1 / 2} \nabla L^{-1}$ and $\nabla^{2} L^{-1}$ on the Musielak-Orlicz-Hardy space $H_{\varphi, L}\left(\mathbb{R}^{n}\right)$ associated with $L$.

On the other hand, it is well known that the variable Lebesgue space $L^{p(\cdot)}\left(\mathbb{R}^{n}\right)$ is a generalization of classical Lebesgue space, via replacing the constant exponent $p$ by a variable exponent function $p(\cdot): \mathbb{R}^{n} \rightarrow(0, \infty)$, which consists of all measurable functions $f$ on $\mathbb{R}^{n}$ such that, for some $\lambda \in(0, \infty), \int_{\mathbb{R}^{n}}[|f(x)| / \lambda]^{p(x)} d x<\infty$, equipped with the Luxemburg (or known as the Luxemburg-Nakano) (quasi-)norm

$$
\|f\|_{L^{p(\cdot)\left(\mathbb{R}^{n}\right)}}:=\inf \left\{\lambda \in(0, \infty): \int_{\mathbb{R}^{n}}\left[\frac{|f(x)|}{\lambda}\right]^{p(x)} d x \leq 1\right\} .
$$

The study of $L^{p}\left(\mathbb{R}^{n}\right)$ originated from Orlicz [19] in 1931. Since then, much attention are paid to the study of variable function spaces. For a detailed history of this topic, we refer the reader to the monographs [5, 8]. As a generalized of the classical Hardy space, the variable Hardy space $H^{p(\cdot)}\left(\mathbb{R}^{n}\right)$ is naturally considered and becomes an active research topic in harmonic analysis, see, for example, [7, 18, 20]. Very recently, Yang et al. [26, 28] studied the variable Hardy spaces associated with different operators.

Let $L=-\operatorname{div}(A \nabla)+V$ be as in (1.1), where $V$ is a nonnegative potential on $\mathbb{R}^{n}$ with $n \geq 3$ and belongs to the reverse Hölder class $R H_{q}\left(\mathbb{R}^{n}\right)$ for some $q \in$ $(n / 2, \infty)$. In this article, motivated by [16, 25, 26], we consider the boundedness of $V L^{-1}, V^{1 / 2} \nabla L^{-1}$ and $\nabla^{2} L^{-1}$ on variable Lebesgue spaces $L^{p(\cdot)}\left(\mathbb{R}^{n}\right)$ and variable Hardy spaces $H_{L}^{p(\cdot)}\left(\mathbb{R}^{n}\right.$ ) associated with $L$ (see Definition 1.6 below). To state the main results, we first recall some notation and definitions.

Let $\mathcal{P}\left(\mathbb{R}^{n}\right)$ be the set of all the measurable functions $p(\cdot): \mathbb{R}^{n} \rightarrow(0, \infty)$ satisfying

$$
p_{-}:=\operatorname{essinf}_{x \in \mathbb{R}^{n}} p(x)>0 \text { and } p_{+}:=\operatorname{essiup}_{x \in \mathbb{R}^{n}} p(x)<\infty .
$$

A function $p(\cdot) \in \mathcal{P}\left(\mathbb{R}^{n}\right)$ is called a variable exponent function on $\mathbb{R}^{n}$. For any $p(\cdot) \in \mathcal{P}\left(\mathbb{R}^{n}\right)$ with $p_{-} \in(1, \infty)$, we define $p^{\prime}(\cdot) \in \mathcal{P}\left(\mathbb{R}^{n}\right)$ by

$$
\frac{1}{p(x)}+\frac{1}{p^{\prime}(x)}=1 \quad \text { for all } x \in \mathbb{R}^{n}
$$

The function $p^{\prime}$ is called the dual variable exponent of $p$.

Recall that a measurable function $g \in \mathcal{P}\left(\mathbb{R}^{n}\right)$ is said to be globally log-Hölder continuous, denoted by $g \in C^{\log }\left(\mathbb{R}^{n}\right)$, if there exist constants $C_{1}, C_{2} \in(0, \infty)$ and $g_{\infty} \in \mathbb{R}$ such that, for any $x, y \in \mathbb{R}^{n}$,

$$
|g(x)-g(y)| \leq \frac{C_{1}}{\log (e+1 /|x-y|)}
$$

and

$$
\left|g(x)-g_{\infty}\right| \leq \frac{C_{2}}{\log (e+|x|)}
$$


The following theorem is the first main result of this article, which establishes the boundedness of $V L^{-1}, V^{1 / 2} \nabla L^{-1}$ and $\nabla^{2} L^{-1}$ on $L^{p(\cdot)}\left(\mathbb{R}^{n}\right)$.

Theorem 1.4. Let $p(\cdot) \in C^{\log }\left(\mathbb{R}^{n}\right), L$ be as in (1.1) and $V \in R H_{q}\left(\mathbb{R}^{n}\right)$ with $q \in(n / 2, \infty)$.

(i) Assume that A satisfies Assumption 1.1. If $1<p_{-} \leq p_{+}<q$, then there exists a positive constant $C$ such that, for any $f \in L^{p(\cdot)}\left(\mathbb{R}^{n}\right)$,

$$
\left\|V L^{-1}(f)\right\|_{L^{p(\cdot)}\left(\mathbb{R}^{n}\right)} \leq C\|f\|_{L^{p(\cdot)}\left(\mathbb{R}^{n}\right)} .
$$

(ii) Assume that $A$ satisfies Assumptions 1.1 and 1.2. If $1<p_{-} \leq p_{+}<p_{0}$, where

$$
p_{0}:= \begin{cases}\frac{2 q n}{3 n-2 q} & \text { if } q \in(n / 2, n) ; \\ 2 q & \text { if } q \in[n, \infty),\end{cases}
$$

then there exists a positive constant $C$ such that, for any $f \in L^{p(\cdot)}\left(\mathbb{R}^{n}\right)$,

$$
\left\|V^{1 / 2} \nabla L^{-1}(f)\right\|_{L^{p(\cdot)\left(\mathbb{R}^{n}\right)}} \leq C\|f\|_{L^{p(\cdot)\left(\mathbb{R}^{n}\right)}} .
$$

(iii) Assume that $A$ satisfies Assumptions 1.1, 1.2 and 1.3. If $1<p_{-} \leq p_{+}<$ $q$, then there exists a positive constant $C$ such that, for any $f \in L^{p(\cdot)}\left(\mathbb{R}^{n}\right)$,

$$
\left\|\nabla^{2} L^{-1}(f)\right\|_{L^{p(\cdot)\left(\mathbb{R}^{n}\right)}} \leq C\|f\|_{L^{p(\cdot)\left(\mathbb{R}^{n}\right)}} .
$$

Remark 1.5. In particular, if $p(\cdot) \equiv p$ is a constant exponent, then Theorem 1.4 coincides with [25, Lemmas 3.2 and 3.3].

The proof of Theorem 1.4 is in Subsection 3.1. We prove it by making use of some known results in [15], the fact that the Hardy-Littlewood operator is bounded on $L^{p(\cdot)}\left(\mathbb{R}^{n}\right)$ (see Lemma 2.6 below) and the extrapolation theorem for $L^{p(\cdot)}\left(\mathbb{R}^{n}\right)$ (see Lemma 3.1 below).

The quadratic operator $S_{L}$, associated to $L$, is defined by setting, for any $f \in$ $L^{2}\left(\mathbb{R}^{n}\right)$ and $x \in \mathbb{R}^{n}$,

$$
S_{L}(f)(x):=\left[\iint_{\Gamma(x)}\left|t^{2} L e^{-t^{2} L}(f)(y)\right|^{2} \frac{d y d t}{t^{n+1}}\right]^{1 / 2}
$$

where $\Gamma(x):=\left\{(y, t) \in \mathbb{R}^{n} \times(0, \infty):|y-x|<t\right\}$ denotes the cone with vertex $x \in \mathbb{R}^{n}$.

Definition 1.6. Let $p(\cdot) \in \mathcal{P}\left(\mathbb{R}^{n}\right)$ satisfy $p_{+} \in(0,1]$ and $L$ be an operator as in (1.1). The variable Hardy space $H_{L}^{p(\cdot)}\left(\mathbb{R}^{n}\right)$ is defined as the completion of the space

$$
\left\{f \in L^{2}\left(\mathbb{R}^{n}\right):\left\|S_{L}(f)\right\|_{L^{p(\cdot)\left(\mathbb{R}^{n}\right)}}<\infty\right\}
$$

with respect to the quasi-norm $\|f\|_{H_{L}^{p(\cdot)}\left(\mathbb{R}^{n}\right)}:=\left\|S_{L}(f)\right\|_{L^{p(\cdot)}\left(\mathbb{R}^{n}\right)}$.

The following theorem establishes the boundedness of $V L^{-1}, V^{1 / 2} \nabla L^{-1}$ and $\nabla^{2} L^{-1}$ on $H_{L}^{p(\cdot)}\left(\mathbb{R}^{n}\right)$. 
Theorem 1.7. Let $L$ be as in (1.1) with A satisfying Assumptions 1.1, 1.2 and 1.3. Assume that $V \in R H_{q}\left(\mathbb{R}^{n}\right)$ with $q \in(\max \{n / 2,2\}, \infty)$ and $p(\cdot) \in C^{\log }\left(\mathbb{R}^{n}\right)$. If $0<p_{-} \leq p_{+} \leq 1$, then there exists a positive constant $C$ such that, for any $f \in H_{L}^{p(\cdot)}\left(\mathbb{R}^{n}\right)$,

$$
\begin{gathered}
\left\|V L^{-1}(f)\right\|_{L^{p(\cdot)}\left(\mathbb{R}^{n}\right)} \leq C\|f\|_{H_{L}^{p(\cdot)}\left(\mathbb{R}^{n}\right)}, \\
\left\|V^{1 / 2} \nabla L^{-1}(f)\right\|_{L^{p(\cdot)}\left(\mathbb{R}^{n}\right)} \leq C\|f\|_{H_{L}^{p(\cdot)}\left(\mathbb{R}^{n}\right)}
\end{gathered}
$$

and

$$
\left\|\nabla^{2} L^{-1}(f)\right\|_{L^{p(\cdot)}\left(\mathbb{R}^{n}\right)} \leq C\|f\|_{H_{L}^{p(\cdot)}\left(\mathbb{R}^{n}\right)} .
$$

Remark 1.8. (i) Noticing that $n \geq 3$, we point out that the assumption $q \in$ $(\max \{n / 2,2\}, \infty)$ guarantees that $q>2$ and $p_{0}>2$, where $p_{0}$ is as in (1.6). By this and Theorem 1.4 (or [25, Lemmas 3.2 and 3.3]), we know that $V L^{-1}, V^{1 / 2} \nabla L^{-1}$ and $\nabla^{2} L^{-1}$ are all bounded on $L^{2}\left(\mathbb{R}^{n}\right)$. Based on this, we prove Theorem 1.7 by estimating these operators acting on the single atom appearing in the atomic decomposition of $H_{L}^{p(\cdot)}\left(\mathbb{R}^{n}\right)$ (see (3.22) below). Observing that the atomic sums in (3.22) converge in $L^{2}\left(\mathbb{R}^{n}\right)$, this is why we assume $q \in(\max \{n / 2,2\}, \infty)$ here.

(ii) When $p(\cdot)=p \in(0,1]$ is a constant exponent and $L=-\Delta+V$ is the Schrödinger operator, (1.9) and (1.11) coincide with [16, Theorem 1.2(a)]. However, (1.10) is new.

The proof of Theorem 1.7 is in Subsection 3.2. We prove it by borrowing some ideas from $[16,17]$ and using some skills from [26]. The key to the proof is to establish some weighted estimates of the spatial derivatives of the heat kernel of $\left\{e^{-t L}\right\}_{t \geq 0}$ (see Lemma 3.6 below). The proof of Lemma 3.6 relies on the upper bound of the heat kernel of $\left\{e^{-t L}\right\}_{t \geq 0}$ (see Lemma 2.3 below) and the inequality (3.4) in Lemma 3.3, which is, in a sense, based on the known $L^{p}\left(\mathbb{R}^{n}\right)$ boundedness of $\nabla^{2} L_{0}^{-1}$ with $L_{0}:=-\operatorname{div}(A \nabla)$ (see [3, Theorem B]). We prove (3.4) by applying the method used in the proof of [17, Lemma 2.7]. However, to overcome the difficulty caused by the elliptic operator $L_{0}=-\operatorname{div}(A \nabla)$ in (3.4), we need to assume that the matrix $A$ satisfies the Assumption 1.3, which plays an essentially key role in the proof of (3.4) as it does in the proof of [3, Theorem $\mathrm{B}]$.

We end this section by making some conventions on notation. In this article, we denote by $C$ a positive constant which is independent of the main parameters, but it may vary from line to line. We also use $C_{(\alpha, \beta, \ldots)}$ to denote a positive constant depending on the parameters $\alpha, \beta, \ldots$ The symbol $f \lesssim g$ means that $f \leq C g$. If $f \lesssim g$ and $g \lesssim f$, we then write $f \sim g$. Let $\mathbb{N}:=\{1,2, \ldots\}, \mathbb{Z}_{+}:=\mathbb{N} \cup\{0\}$ and $\overrightarrow{0}_{n}:=(0, \ldots, 0) \in \mathbb{R}^{n}$. For any measurable subset $E$ of $\mathbb{R}^{n}$, we denote by $E^{\complement}$ the set $\mathbb{R}^{n} \backslash E$. For any $p \in(0, \infty)$ and any measurable subset $E$ of $\mathbb{R}^{n}$, let $L^{p}(E)$ be the set of all measurable functions $f$ on $\mathbb{R}^{n}$ such that

$$
\|f\|_{L^{p}(E)}:=\left[\int_{E}|f(x)|^{p} d x\right]^{1 / p}<\infty .
$$


For any $k \in \mathbb{N}$ and $p \in(1, \infty)$, denote by $W^{k, p}\left(\mathbb{R}^{n}\right)$ the usual Sobolev space on $\mathbb{R}^{n}$ equipped with the norm $\left(\sum_{|\alpha| \leq k}\left\|D^{\alpha} f\right\|_{L^{p}\left(\mathbb{R}^{n}\right)}^{p}\right)^{1 / p}$, where $\alpha \in \mathbb{Z}_{+}^{n}$ is a multiindex and $D^{\alpha} f$ denotes the distributional derivatives of $f$. We also denote by $C_{c}^{\infty}\left(\mathbb{R}^{n}\right)$ the set of all infinitely differential functions with compact supports. For any $r \in \mathbb{R}$, the symbol $\lfloor r\rfloor$ denotes the largest integer $m$ such that $m \leq r$. For any $\mu \in(0, \pi)$, let

$$
\Sigma_{\mu}^{0}:=\{z \in \mathbb{C} \backslash\{0\}:|\arg z|<\mu\} .
$$

For any ball $B:=B\left(x_{B}, r_{B}\right):=\left\{y \in \mathbb{R}^{n}:|x-y|<r_{B}\right\} \subset \mathbb{R}^{n}$ with $x_{B} \in \mathbb{R}^{n}$ and $r_{B} \in(0, \infty), \alpha \in(0, \infty)$ and $j \in \mathbb{N}$, we let $\alpha B:=B\left(x_{B}, \alpha r_{B}\right)$,

$$
U_{0}(B):=B \quad \text { and } \quad U_{j}(B):=\left(2^{j} B\right) \backslash\left(2^{j-1} B\right) .
$$

For any $p \in[1, \infty], p^{\prime}$ denotes its conjugate number, namely, $1 / p+1 / p^{\prime}=1$.

\section{Preliminaries}

In this section, we recall some notions and results on the Schrödinger type operator $L=-\operatorname{div}(A \nabla)+V$ and variable Lebesgue space $L^{p(\cdot)}\left(\mathbb{R}^{n}\right)$.

We first recall the definition of the auxiliary function $m(\cdot, V)$ introduced by Shen [21, Definition 2.1] and its properties. Let $V \in R H_{q}\left(\mathbb{R}^{n}\right), q \in(n / 2, \infty)$, and $V \not \equiv 0$. For any $x \in \mathbb{R}^{n}$, the auxiliary function $m(x, V)$ is defined by

$$
\frac{1}{m(x, V)}:=\sup \left\{r \in(0, \infty): \frac{1}{r^{n-2}} \int_{B(x, r)} V(y) d y \leq 1\right\} .
$$

For the auxiliary function $m(\cdot, V)$, we have the following Lemma 2.1, which is proved in [21, Lemmas 1.2 and 1.8].

Lemma 2.1 ([21]). Let $m(\cdot, V)$ be as in (2.1) and $V \in R H_{q}\left(\mathbb{R}^{n}\right)$ with $q \in$ $(n / 2, \infty)$.

(i) Then there exist a positive constant $C$ such that, for any $x \in \mathbb{R}^{n}$ and $0<r<R<\infty$,

$$
\frac{1}{r^{n-2}} \int_{B(x, r)} V(y) d y \leq C\left(\frac{R}{r}\right)^{\frac{n}{q}-2} \frac{1}{R^{n-2}} \int_{B(x, R)} V(y) d y .
$$

(ii) There exist positive constants $C$ and $k_{0}$ such that, for any $x \in \mathbb{R}^{n}$ and $R \in(0, \infty)$, if $\operatorname{Rm}(x, V) \geq 1$, then

$$
\frac{1}{R^{n-2}} \int_{B(x, R)} V(y) d y \leq C[R m(x, V)]^{k_{0}} .
$$

The following lemma is [2, Theorem 4].

Lemma $2.2([2])$. Let $L_{0}:=-\operatorname{div}(A \nabla)$ with $A$ satisfying Assumption 1.1 and $\left\{e^{-t L_{0}}\right\}_{t \geq 0}$ the heat semigroup generated by $L_{0}$. Then the kernels $h_{t}(x, y)$ of the heat semigroup $\left\{e^{-t L_{0}}\right\}_{t \geq 0}$ are continuous and there exists a constant $\alpha_{0} \in(0,1]$ such that, for any given $\alpha \in\left(0, \alpha_{0}\right)$,

$$
\left|h_{t}(x+h, y)-h_{t}(x, y)\right|+\left|h_{t}(x, y+h)-h_{t}(x, y)\right| \leq \frac{C}{t^{n / 2}}\left[\frac{|h|}{\sqrt{t}}\right]^{\alpha} e^{-c \frac{|x-y|^{2}}{t}}
$$


where $t \in(0, \infty), x, y, h \in \mathbb{R}^{n}$ with $|h| \leq \sqrt{t}$ and $C, c$ are positive constants independent of $t, x, y, h$.

The following lemma is [25, Lemma 2.6].

Lemma 2.3 ([25]). Let $L$ be as in (1.1) with A satisfying Assumption 1.1 and $V \in R H_{q}\left(\mathbb{R}^{n}\right), q \in(n / 2, \infty)$. Assume that $K_{t}$ is the kernel of the heat semigroup $\left\{e^{-t L}\right\}_{t \geq 0}$ and let

$$
\mu_{0}:=\min \left\{\alpha_{0}, 2-\frac{n}{q}\right\}
$$

where $\alpha_{0} \in(0,1]$ is as in Lemma 2.2.

(i) For any given $N \in \mathbb{N}$, there exist positive constants $C_{(N)}$ and $c$ such that, for any $t \in(0, \infty)$ and every $(x, y) \in \mathbb{R}^{n} \times \mathbb{R}^{n}$,

$$
0 \leq K_{t}(x, y) \leq \frac{C_{(N)}}{t^{n / 2}} e^{-c \frac{|x-y|^{2}}{t}}[1+\sqrt{t} m(x, V)+\sqrt{t} m(y, V)]^{-N} .
$$

(ii) For any given $N \in \mathbb{N}$ and $\mu \in\left(0, \mu_{0}\right)$, there exist positive constants $C_{(N, \mu)}$ and $c$ such that, for any $t \in(0, \infty)$ and every $x, y, h \in \mathbb{R}^{n}$ with $|h| \leq \sqrt{t}$,

$$
\begin{aligned}
& \left|K_{t}(x+h, y)-K_{t}(x, y)\right|+\left|K_{t}(x, y+h)-K_{t}(x, y)\right| \\
& \quad \leq \frac{C_{(N, \mu)}}{t^{n / 2}}\left[\frac{|h|}{\sqrt{t}}\right]^{\mu} e^{-c \frac{|x-y|^{2}}{t}}[1+\sqrt{t} m(x, V)+\sqrt{t} m(y, V)]^{-N} .
\end{aligned}
$$

Remark 2.4. (i) For any given $N \in \mathbb{N}$, by an argument similar to that used in the proof of [11, Corollary 6.4], we find that, for any $z \in \Sigma_{\pi / 5}^{0}$,

$$
\left|K_{z}(x, y)\right| \leq C_{(N)} \frac{1}{|z|^{n / 2}} e^{-c \frac{|x-y|^{2}}{|z|}}[1+\sqrt{|z|} m(x, V)+\sqrt{|z|} m(y, V)]^{-N}
$$

where $K_{z}(\cdot, \cdot)$ denotes the integral kernel of the operator $e^{-z L}$ and $C_{(N)}$ is a positive constant depending only on $N$. From the Cauchy formula and the holomorphy of the semigroup $\left\{e^{-z L}\right\}_{z \in \Sigma_{\pi / 5}^{0}}$, we deduce that, for any $k \in \mathbb{N}$ and $t \in(0, \infty)$,

$$
\frac{d^{k}}{d t^{k}} e^{-t L}=(-L)^{k} e^{-t L}=\frac{k !}{2 \pi i} \int_{|\zeta-t|=\eta t} \frac{e^{-\zeta L}}{(\zeta-t)^{k+1}} d \zeta
$$

where $\eta \in(0, \infty)$ is small enough such that $\{\zeta \in \mathbb{C}:|\zeta-t|=\eta t\} \varsubsetneqq \Sigma_{\pi / 5}^{0}$ and $\Sigma_{\pi / 5}^{0}$ is as in (1.12). Hence, for every $x, y \in \mathbb{R}^{n}$, we have

$$
\frac{\partial^{k}}{\partial t^{k}} K_{t}(x, y)=(-1)^{k} \frac{k !}{2 \pi i} \int_{|\zeta-t|=\eta t} \frac{K_{\zeta}(x, y)}{(\zeta-t)^{k+1}} d \zeta
$$

From this, (2.5) and the fact that $|\zeta| \sim t$ for any $\zeta \in\{\zeta \in \mathbb{C}:|\zeta-t|=\eta t\}$, it follows that

$$
\begin{aligned}
\left|\frac{\partial^{k}}{\partial t^{k}} K_{t}(x, y)\right| & \lesssim \int_{|\zeta-t|=\eta t} \frac{\left|K_{\zeta}(x, y)\right|}{|\zeta-t|^{k+1}} d|\zeta| \\
& \lesssim \frac{1}{t^{k+n / 2}} e^{-c \frac{|x-y|^{2}}{t}}[1+m(x, V)+m(y, V)]^{-N}
\end{aligned}
$$


where the implicit positive constant depends only on $k, \eta$ and $N$.

(ii) For any given $N \in \mathbb{N}$ and $\mu \in\left(0, \mu_{0}\right)$, by [2, Lemma 17], we know that, for any $z \in \Sigma_{\pi / 5}^{0}$ and $y \in \mathbb{R}^{n}$,

$$
\left\|K_{z}(\cdot, y)\right\|_{C^{\mu}\left(\mathbb{R}^{n}\right)} \lesssim|z|^{-\frac{n+\mu}{2}}
$$

is equivalent to, for any $f \in L^{1}\left(\mathbb{R}^{n}\right)$,

$$
\left\|e^{-z L}(f)\right\|_{C^{\mu}\left(\mathbb{R}^{n}\right)} \lesssim|z|^{-\frac{n+\mu}{2}}\|f\|_{L^{1}\left(\mathbb{R}^{n}\right)} .
$$

Similar to the proof of [2, Lemma 19], by (2.4) and interpolation, we obtain (2.9), namely, (2.8) holds true. From (2.8) and an argument similar to the proof [11, Proposition 4.11], we further deduce that, for any given $N \in \mathbb{N}$, there exists positive constants $C_{(N, \mu)}$ and $c$ such that, for any $z \in \Sigma_{\pi / 5}^{0}$ and every $x, y, h \in \mathbb{R}^{n}$ with $|h| \leq \sqrt{|z|}$,

$$
\begin{aligned}
& \left|K_{z}(x+h, y)-K_{t}(x, y)\right|+\left|K_{z}(x, y+h)-K_{t}(x, y)\right| \\
& \quad \leq \frac{C_{(N, \mu)}}{|z|^{n / 2}}\left[\frac{|h|}{\sqrt{|z|}}\right]^{\mu} e^{-c \frac{|x-y|^{2}}{|z|}}[1+\sqrt{|z|} m(x, V)+\sqrt{|z|} m(y, V)]^{-N} .
\end{aligned}
$$

This, combined with (2.6), implies that, for any $k \in \mathbb{N}$,

$$
\begin{aligned}
& \left|\frac{\partial^{k}}{\partial t^{k}} K_{t}(x+h, y)-\frac{\partial^{k}}{\partial t^{k}} K_{t}(x, y)\right|+\left|\frac{\partial^{k}}{\partial t^{k}} K_{t}(x, y+h)-\frac{\partial^{k}}{\partial t^{k}} K_{t}(x, y)\right| \\
& \leq \frac{C_{(N, \mu)}}{t^{k+n / 2}}\left[\frac{|h|}{\sqrt{t}}\right]^{\mu} e^{-c \frac{|x-y|^{2}}{t}}[1+\sqrt{t} m(x, V)+\sqrt{t} m(y, V)]^{-N} .
\end{aligned}
$$

Remark 2.5. By Lemma 2.3(i), we know that the heat kernel $K_{t}$ satisfies the Gaussian upper bound. From this and [24, (3.2)], we deduce that, for any $p \in$ $(1, \infty)$, the quadratic operator $S_{L}$ (see $\left.(1.8)\right)$ is bounded on $L^{p}\left(\mathbb{R}^{n}\right)$.

The Hardy-Littlewood maximal operator $\mathcal{M}$ is defined by setting, for any $f \in$ $L_{\text {loc }}^{1}\left(\mathbb{R}^{n}\right)$ and $x \in \mathbb{R}^{n}$,

$$
\mathcal{M}(f)(x):=\sup _{B \ni x} \frac{1}{|B|} \int_{B}|f(y)| d y,
$$

where the supremum is taken over all balls $B$ of $\mathbb{R}^{n}$ containing $x$.

The following lemma establishes the boundedness of $\mathcal{M}$ on $L^{p(\cdot)}\left(\mathbb{R}^{n}\right)$, which is just [8, Theorem 4.3.8] (see also [5, Theorem 3.16]).

Lemma $2.6([8])$. Let $p(\cdot) \in C^{\log }\left(\mathbb{R}^{n}\right)$ and $1<p_{-} \leq p_{+}<\infty$. Then there exists a positive constant $C$ such that, for any $f \in L^{p(\cdot)}\left(\mathbb{R}^{n}\right)$,

$$
\|\mathcal{M}(f)\|_{L^{p(\cdot)\left(\mathbb{R}^{n}\right)}} \leq C\|f\|_{L^{p(\cdot)}\left(\mathbb{R}^{n}\right)} .
$$

The following lemma is a particular case of [26, Lemma 2.4], which is is a slight variant of [20, Lemma 4.1].

Lemma $2.7([26])$. Let $\kappa \in[1, \infty), p(\cdot) \in C^{\log }\left(\mathbb{R}^{n}\right), \underline{p}:=\min \left\{p_{-}, 1\right\}$ and $r \in$ $[1, \infty] \cap\left(p_{+}, \infty\right]$, where $p_{-}$and $p_{+}$are as in (1.3). Then there exists a positive constant $C$ such that, for any sequence $\left\{B_{j}\right\}_{j \in \mathbb{N}}$ of balls in $\mathbb{R}^{n},\left\{\lambda_{j}\right\}_{j \in \mathbb{N}} \subset \mathbb{C}$ and 
functions $\left\{a_{j}\right\}_{j \in \mathbb{N}}$ satisfying that, for any $j \in \mathbb{N}$, supp $a_{j} \subset \kappa B_{j}$ and $\left\|a_{j}\right\|_{L^{r}\left(\mathbb{R}^{n}\right)} \leq$ $\left|B_{j}\right|^{1 / r}$,

$$
\left\|\left(\sum_{j=1}^{\infty}\left|\lambda_{j} a_{j}\right|^{\underline{p}}\right)^{\underline{\underline{p}}}\right\|_{L^{p(\cdot)\left(\mathbb{R}^{n}\right)}} \leq C \kappa^{n\left(\underline{\underline{\underline{p}}}-\frac{1}{r}\right)}\left\|\left(\sum_{j=1}^{\infty}\left|\lambda_{j} \chi_{B_{j}}\right| \underline{\underline{p}}\right)^{\underline{\underline{p}}}\right\|_{L^{p(\cdot)}\left(\mathbb{R}^{n}\right)} .
$$

For more properties of the variable Lebesgue spaces $L^{p(\cdot)}\left(\mathbb{R}^{n}\right)$, we refer the reader to $[5,8]$.

Remark 2.8. Let $p(\cdot) \in \mathcal{P}\left(\mathbb{R}^{n}\right)$.

(i) For any $\lambda \in \mathbb{C}$ and $f \in L^{p(\cdot)}\left(\mathbb{R}^{n}\right),\|\lambda f\|_{L^{p(\cdot)}\left(\mathbb{R}^{n}\right)}=|\lambda|\|f\|_{L^{p(\cdot)}\left(\mathbb{R}^{n}\right)}$. In particular, if $p_{-} \in[1, \infty)$, then $\|\cdot\|_{L^{p(\cdot)}\left(\mathbb{R}^{n}\right)}$ is a norm, namely, for any $f, g \in L^{p(\cdot)}\left(\mathbb{R}^{n}\right)$,

$$
\|f+g\|_{L^{p(\cdot)}\left(\mathbb{R}^{n}\right)} \leq\|f\|_{L^{p(\cdot)}\left(\mathbb{R}^{n}\right)}+\|g\|_{L^{p(\cdot)}\left(\mathbb{R}^{n}\right)} .
$$

(ii) By the definition of $\|\cdot\|_{L^{p(\cdot)}\left(\mathbb{R}^{n}\right)}$ (see (1.2)), it is easy to see that, for any $f \in L^{p(\cdot)}\left(\mathbb{R}^{n}\right)$ and $s \in(0, \infty)$,

$$
\left\||f|^{s}\right\|_{L^{p(\cdot)\left(\mathbb{R}^{n}\right)}}=\|f\|_{L^{s p(\cdot)\left(\mathbb{R}^{n}\right)}}^{s} .
$$

(iii) Let $p_{-} \in(1, \infty)$. Then, by $[8$, Lemma 3.2.20], we find that, for any $f \in L^{p(\cdot)}\left(\mathbb{R}^{n}\right)$ and $g \in L^{p^{\prime}(\cdot)}\left(\mathbb{R}^{n}\right)$,

$$
\int_{\mathbb{R}^{n}}|f(x) g(x)| d x \leq 2\|f\|_{L^{p(\cdot)}\left(\mathbb{R}^{n}\right)}\|g\|_{L^{p^{\prime}(\cdot)\left(\mathbb{R}^{n}\right)}},
$$

where $p^{\prime}(\cdot)$ is the dual variable exponent of $p(\cdot)$, which is defined in (1.4).

(iv) Let $p_{-} \in(1, \infty)$. Then, by $[5$, Theorem 2.34] and [8, Corollary 3.2.14]), we know that, for any $f \in L^{p(\cdot)}\left(\mathbb{R}^{n}\right)$,

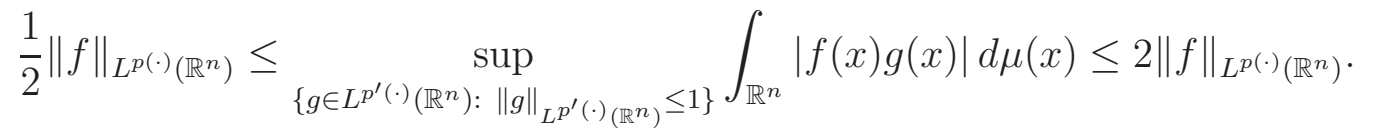

\section{Proofs of MAin Results}

3.1. Proof of Theorem 1.4. In this subsection, we prove Theorem 1.4. We begin with introducing some auxiliary lemmas.

Let $q \in[1, \infty)$. Recall that a non-negative and locally integrable function $w$ on $\mathbb{R}^{n}$ is said to belong to the class $A_{q}\left(\mathbb{R}^{n}\right)$ of Muckenhoupt weights, denoted by $w \in A_{q}\left(\mathbb{R}^{n}\right)$, if, when $q \in(1, \infty)$,

or

$$
A_{q}(w):=\sup _{B \subset \mathbb{R}^{n}} \frac{1}{|B|} \int_{B} w(x) d x\left\{\frac{1}{|B|} \int_{B}[w(x)]^{-\frac{1}{q-1}} d x\right\}^{q-1}<\infty
$$

$$
A_{1}(w):=\sup _{B \subset \mathbb{R}^{n}} \frac{1}{|B|} \int_{B} w(x) d x\left\{\operatorname{essinf}_{x \in B} w(x)\right\}^{-1}<\infty
$$

where the suprema are taken over all balls $B$ of $\mathbb{R}^{n}$.

We need the following lemma which is called the extrapolation theorem for $L^{p(\cdot)}\left(\mathbb{R}^{n}\right)$ (see, for example, [6, Theorem 1.3]). 
Lemma 3.1 ([6]). Let $\mathcal{F}$ be a family of pairs of measurable functions on $\mathbb{R}^{n}$. Assume that, for some $p_{0} \in(0, \infty)$ and any $w \in A_{1}\left(\mathbb{R}^{n}\right)$,

$$
\int_{\mathbb{R}^{n}}|f(x)|^{p_{0}} w(x) d x \leq C_{(w)} \int_{\mathbb{R}^{n}}|g(x)|^{p_{0}} w(x) d x \quad \text { for any } \quad(f, g) \in \mathcal{F}
$$

where the positive constant $C_{(w)}$ depends only on $A_{1}(w)$. Let $p(\cdot) \in C^{\log }\left(\mathbb{R}^{n}\right)$ such that $p_{-} \in\left(p_{0}, \infty\right)$. Then there exists a positive constant $C$ such that, for any $(f, g) \in \mathcal{F}$,

$$
\|f\|_{L^{p(\cdot)}\left(\mathbb{R}^{n}\right)} \leq C\|g\|_{L^{p(\cdot)}\left(\mathbb{R}^{n}\right)}
$$

We also need the following lemma, which is just [15, Theorem 1.7] and plays a key role in the proof of Theorem 1.4.

Lemma $3.2([15])$. Let $L$ be as in $(1.1)$ and $\left(V L^{-1}\right)^{*},\left(V^{1 / 2} \nabla L^{-1}\right)^{*}$ the usual dual operators of $V L^{-1}, V^{1 / 2} \nabla L^{-1}$.

(i) If A satisfies Assumption 1.1 and $V \in R H_{q}\left(\mathbb{R}^{n}\right)$ with $q \in(n / 2, \infty)$, then there exists a positive constant $C$ such that, for any $f \in C_{c}^{\infty}\left(\mathbb{R}^{n}\right)$ and $x \in \mathbb{R}^{n}$,

$$
\left|\left(V L^{-1}\right)^{*}(f)(x)\right| \leq C\left[\mathcal{M}\left(|f|^{q^{\prime}}\right)(x)\right]^{1 / q^{\prime}}
$$

where $\mathcal{M}$ is the Hardy-Littlewood maximal operator as in (2.11) and $q^{\prime}$ the conjugate number of $q$.

(ii) If $A$ satisfies Assumptions 1.1 and 1.2, and $V \in R H_{q}\left(\mathbb{R}^{n}\right)$ with $q \in$ $(n / 2, \infty)$, then there exists a positive constant $C$ such that, for any $f \in$ $C_{c}^{\infty}\left(\mathbb{R}^{n}\right)$ and $x \in \mathbb{R}^{n}$,

$$
\left|\left(V^{1 / 2} \nabla L^{-1}\right)^{*}(f)(x)\right| \leq C\left[\mathcal{M}\left(|f|^{p_{0}^{\prime}}\right)(x)\right]^{1 / p_{0}^{\prime}},
$$

where $p_{0}$ is as in (1.6) and $p_{0}^{\prime}$ the conjugate number of $p_{0}$.

We are now in a position to prove Theorem 1.4.

Proof of Theorem 1.4. We first prove (i). For any $f, g \in C_{c}^{\infty}\left(\mathbb{R}^{n}\right)$, we have

$$
\begin{aligned}
\left\langle V L^{-1}(f), g\right\rangle & :=\int_{\mathbb{R}^{n}} V L^{-1}(f)(x) g(x) d x \\
& =\int_{\mathbb{R}^{n}}\left[\int_{\mathbb{R}^{n}} V(x) \Gamma(x, y) f(y) d y\right] g(x) d x \\
& =\int_{\mathbb{R}^{n}} f(y)\left[\int_{\mathbb{R}^{n}} V(x) \Gamma(x, y) g(x) d x\right] d y \\
& =:\left\langle f,\left(V L^{-1}\right)^{*}(g)\right\rangle,
\end{aligned}
$$


where $\Gamma(\cdot, \cdot)$ denotes the fundamental solution of $L$. By (3.1) and Remark 2.8(iv), we have

$$
\begin{aligned}
& \left\|V L^{-1}(f)\right\|_{L^{p(\cdot)\left(\mathbb{R}^{n}\right)}} \lesssim \sup _{\|g\|_{L^{p^{\prime}(\cdot)}\left(\mathbb{R}^{n}\right)} \leq 1}\left|\int_{\mathbb{R}^{n}} V L^{-1}(f)(x) g(x) d x\right| \\
& \sim \sup _{\|g\|_{L^{p^{\prime}(\cdot)}\left(\mathbb{R}^{n}\right)} \leq 1}\left|\int_{\mathbb{R}^{n}} f(x)\left(V L^{-1}\right)^{*}(g)(x) d x\right| \\
& \lesssim \sup _{\|g\|_{L^{p^{\prime}(\cdot)}\left(\mathbb{R}^{n}\right)} \leq 1}\|f\|_{L^{p(\cdot)}\left(\mathbb{R}^{n}\right)}\left\|\left(V L^{-1}\right)^{*}(g)\right\|_{L^{p^{\prime}(\cdot)\left(\mathbb{R}^{n}\right)}} .
\end{aligned}
$$

By Lemma 3.2(i), we know that, for any $f \in C_{c}^{\infty}\left(\mathbb{R}^{n}\right)$ and $x \in \mathbb{R}^{n}$,

$$
\left|\left(V L^{-1}\right)^{*}(f)(x)\right| \lesssim\left[M\left(|f|^{q^{\prime}}\right)(x)\right]^{1 / q^{\prime}} .
$$

Moreover, by the fact that $1<p_{-} \leq p_{+}<q$, it is easy to see that $1<q^{\prime}<p_{-}^{\prime} \leq$ $p_{+}^{\prime}<\infty$. Hence, $\left(\frac{p^{\prime}(\cdot)}{q^{\prime}}\right)_{-}>1$. From this, (3.3), Remark 2.8(ii) and Lemma 2.6, we deduce that, for any $g \in C_{c}^{\infty}\left(\mathbb{R}^{n}\right)$,

$$
\begin{aligned}
\left\|\left(V L^{-1}\right)^{*}(g)\right\|_{L^{p^{\prime}(\cdot)\left(\mathbb{R}^{n}\right)}} & \lesssim\left\|\left[M\left(|g|^{q^{\prime}}\right)\right]^{1 / q^{\prime}}\right\|_{L^{p^{\prime}(\cdot)\left(\mathbb{R}^{n}\right)}} \sim\left\|M\left(|g|^{q^{\prime}}\right)\right\|_{L^{\frac{p^{\prime}(\cdot)}{q^{\prime}}}\left(\mathbb{R}^{n}\right)}^{1 / q^{\prime}} \\
& \lesssim\left\||g|^{q^{\prime}}\right\|_{L^{\frac{p^{\prime}(\cdot)}{q^{\prime}}\left(\mathbb{R}^{n}\right)}}^{1 / q^{\prime}} \sim\|g\|_{L^{p^{\prime}(\cdot)\left(\mathbb{R}^{n}\right)}}
\end{aligned}
$$

From this and (3.2), we deduce that (1.5) holds true.

For (ii), by Lemma 3.2(ii) and an argument similar to that used in the proof of (i), we know that (1.7) holds true.

Next, we prove (iii). Let $L_{0}:=-\operatorname{div}(A \nabla)=L-V$. Then, by [15, Theorem 2.7], we find that, for any given $p \in(1, \infty)$ and $w \in A_{p}\left(\mathbb{R}^{n}\right), \nabla^{2} L_{0}^{-1}$ is bounded on the weighted Lebesgue space $L^{p}(w)$, which is defined to be the set of all measurable function $f$ on $\mathbb{R}^{n}$ such that

$$
\|f\|_{L^{p}(w)}:=\left[\int_{\mathbb{R}^{n}}|f(x)|^{p} w(x) d x\right]^{1 / p}<\infty .
$$

From this and Lemma 3.1, we deduce that if $1<p_{-} \leq p_{+}<\infty$, then $\nabla^{2} L_{0}^{-1}$ is bounded on $L^{p(\cdot)}\left(\mathbb{R}^{n}\right)$. By this, (1.5) and the fact that $L=L_{0}+V$, we find that if $1<p_{-} \leq p_{+}<q$, then, for any $f \in L^{p(\cdot)}\left(\mathbb{R}^{n}\right)$,

$$
\begin{aligned}
\left\|\nabla^{2} L^{-1}(f)\right\|_{L^{p(\cdot)}\left(\mathbb{R}^{n}\right)} & =\left\|\nabla^{2} L_{0}^{-1} L_{0} L^{-1}\right\|_{L^{p(\cdot)\left(\mathbb{R}^{n}\right)}} \\
& \lesssim\left\|(L-V) L^{-1}(f)\right\|_{L^{p(\cdot)}\left(\mathbb{R}^{n}\right)} \lesssim\|f\|_{L^{p(\cdot)}\left(\mathbb{R}^{n}\right)} .
\end{aligned}
$$

This finishes the proof of Theorem 1.4.

3.2. Proof of Theorem 1.7. In this subsection, we give the proof of Theorem 1.7. To this end, we apply the method used in $[16,17]$. We begin with introducing an inequality, which is an analogue of [17, Lemma 2.7] and inspired by coercive estimates on semiconvex domains established in [9, Theorem 4.8 and Lemma $4.17]$. 
Lemma 3.3. Let $p \in(1, \infty)$ and $L_{0}:=-\operatorname{div}(A \nabla)$ with $A$ satisfying Assumptions 1.1, 1.2 and 1.3. Then there exists a positive constant $C$ such that, for any $f \in W^{2, p}\left(\mathbb{R}^{n}\right)$ and $\phi \in C_{c}^{\infty}\left(\mathbb{R}^{n}\right)$,

$$
\begin{aligned}
& \left\|\phi\left|\nabla^{2} f\right|\right\|_{L^{p}\left(\mathbb{R}^{n}\right)} \\
& \quad \leq C\left[\left\|f\left|\nabla^{2} \phi\right|\right\|_{L^{p}\left(\mathbb{R}^{n}\right)}+\left\|\left|\nabla f\|\nabla \phi \mid\|_{L^{p}\left(\mathbb{R}^{n}\right)}+\left\|\phi L_{0}(f)\right\|_{L^{p}\left(\mathbb{R}^{n}\right)}\right] .\right.\right.
\end{aligned}
$$

Proof. Indeed, for any $f \in W^{2, p}\left(\mathbb{R}^{n}\right)$ and $\phi \in C_{c}^{\infty}\left(\mathbb{R}^{n}\right)$, we have

$$
\begin{aligned}
\phi \partial_{j}\left(\partial_{k} f\right) & =\partial_{j}\left(\phi \partial_{k} f\right)-\partial_{j} \phi \partial_{k} f=\partial_{j}\left(\partial_{k}(\phi f)-\left(\partial_{k} \phi\right) f\right)-\partial_{j} \phi \partial_{k} f \\
& =\partial_{j}\left(\partial_{k}(\phi f)\right)-\partial_{j} f \partial_{k} \phi-f \partial_{j}\left(\partial_{k} \phi\right)-\partial_{j} \phi \partial_{k} f .
\end{aligned}
$$

By this and the fact that $\nabla^{2} L_{0}^{-1}$ is bounded on $L^{p}\left(\mathbb{R}^{n}\right)$ for any $p \in(1, \infty)$ (see [15, Theorem 2.7]), we know that

$$
\begin{aligned}
& \left\|\phi \partial_{j}\left(\partial_{k} f\right)\right\|_{L^{p}\left(\mathbb{R}^{n}\right)} \\
& \quad \leq\left\|\nabla^{2}(\phi f)\right\|_{L^{p}\left(\mathbb{R}^{n}\right)}+2\left\|\left|\nabla f\left\|\nabla \phi\left|\left\|_{L^{p}\left(\mathbb{R}^{n}\right)}+\right\| f\right| \nabla^{2} \phi \mid\right\|_{L^{p}\left(\mathbb{R}^{n}\right)}\right.\right. \\
& \quad \lesssim\left\|L_{0}(\phi f)\right\|_{L^{p}\left(\mathbb{R}^{n}\right)}+2\left\|\left|\nabla f\|\nabla \phi\|\left\|_{L^{p}\left(\mathbb{R}^{n}\right)}+\right\| f\right| \nabla^{2} \phi \mid\right\|_{L^{p}\left(\mathbb{R}^{n}\right)} .
\end{aligned}
$$

For $L_{0}(\phi f)$, it holds true that

$$
\begin{aligned}
L_{0}(\phi f) & =-\operatorname{div}(f(A \nabla \phi)+\phi(A \nabla f)) \\
& =-(A \nabla \phi) \cdot(\nabla f)+f L_{0}(\phi)-(A \nabla f) \cdot(\nabla \phi)+\phi L_{0}(f) .
\end{aligned}
$$

Thus, we obtain

$$
\left\|L_{0}(\phi f)\right\|_{L^{p}\left(\mathbb{R}^{n}\right)} \lesssim\left\|f L_{0}(\phi)\right\|_{L^{p}\left(\mathbb{R}^{n}\right)}+\|\mid \nabla \phi\| \nabla f\left\|_{L^{p}\left(\mathbb{R}^{n}\right)}+\right\| \phi L_{0}(f) \|_{L^{p}\left(\mathbb{R}^{n}\right)} .
$$

For $L_{0}(\phi)$, we find that

$$
\begin{aligned}
L_{0}(\phi) & =-\operatorname{div}(A \nabla \phi)=-\sum_{i=1}^{n} \partial_{i}\left(\sum_{j=1}^{n} a_{i j}\left(\partial_{j} \phi\right)\right) \\
& =-\sum_{i=1}^{n} \sum_{j=1}^{n}\left[\left(\partial_{i} a_{i j}\right)\left(\partial_{j} \phi\right)+a_{i j}\left(\partial_{i} \partial_{j} \phi\right)\right] .
\end{aligned}
$$

By Assumption 1.3, we know that $\sum_{i=1}^{n} \partial_{i} a_{i j}=0$ for any $j \in\{1, \ldots, n\}$. This further implies that $-\sum_{i=1}^{n} \sum_{j=1}^{n}\left(\partial_{i} a_{i j}\right)\left(\partial_{j} \phi\right)=-\sum_{j=1}^{n} \sum_{i=1}^{n}\left(\partial_{i} a_{i j}\right)\left(\partial_{j} \phi\right)=0$. From this, (3.7) and Assumption 1.1, we deduce that

$$
\left|L_{0}(\phi)\right|=\left|\sum_{i=1}^{n} \sum_{j=1}^{n} a_{i j}\left(\partial_{i} \partial_{j} \phi\right)\right| \lesssim\left|\nabla^{2} \phi\right| .
$$

This, combined with (3.5) and (3.6), implies that (3.4) holds true. Hence, we complete the proof of Lemma 3.3.

Remark 3.4. In Lemma 3.3, if $A=I$ is the identity matrix, namely, $L_{0}=-\Delta$ is the Laplacian operator, then Lemma 3.3 is just [17, Lemma 2.7]. 
Next, inspired by F. K. Ly [16, Proposition 3.3] and [17, Proposition 2.4], we introduce some weighted estimates for the spatial derivatives of the heat kernel of $\left\{e^{-t L}\right\}_{t \geq 0}$, which play a key role in the proof of Theorem 1.7.

Lemma 3.5. Assume $V \in R H_{q}\left(\mathbb{R}^{n}\right)$ with $q \in(n / 2, \infty)$. Let $K_{t}$ be the kernel of the heat semigroup $\left\{e^{-t L}\right\}_{t \geq 0}$ and $q^{*}:=\frac{n q}{n-q}$ if $q \in(n / 2, n)$, or $q^{*}:=\infty$ if $q \in[n, \infty]$. For any given $p \in\left[1, q^{*}\right)$ and $N \in \mathbb{N}$, there exist positive constants $\alpha, C$ and $c$ such that, for any $y \in \mathbb{R}^{n}$ and $t \in(0, \infty)$,

$$
\left[\int_{\mathbb{R}^{n}}\left|\nabla_{x} \frac{\partial^{k}}{\partial t^{k}} K_{t}(x, y)\right|^{p} e^{\alpha \frac{|x-y|^{2}}{t}} d x\right]^{1 / p} \leq \frac{C}{t^{1 / 2+k+n / 2 p^{\prime}}}[1+\sqrt{t} m(y, V)]^{-N} \text {. }
$$

The proof of Lemma 3.5 is totally similar to those of [17, Proposition 2.4(b)] [16, Proposition 3.3], the details being omitted.

Lemma 3.6. Let $V \in R H_{q}\left(\mathbb{R}^{n}\right)$ with $q \in(n / 2, \infty)$ and $K_{t}$ the kernel of the heat semigroup $\left\{e^{-t L}\right\}_{t \geq 0}$.

(i) For any given $p \in(1, q], k \in \mathbb{Z}_{+}$and $N \in \mathbb{N}$, there exist positive constants $\alpha$ and $C$ such that, for any $t \in(0, \infty)$ and $y \in \mathbb{R}^{n}$,

$$
\left[\int_{\mathbb{R}^{n}}\left|\nabla_{x}^{2} \frac{\partial^{k}}{\partial t^{k}} K_{t}(x, y)\right|^{p} e^{\alpha \frac{|x-y|^{2}}{t}} d x\right]^{1 / p} \leq \frac{C}{t^{1+k+n / 2 p^{\prime}}}[1+\sqrt{t} m(y, V)]^{-N}
$$

and

$$
\begin{aligned}
{\left[\int_{\mathbb{R}^{n}}\left|V(x) \frac{\partial^{k}}{\partial t^{k}} K_{t}(x, y)\right|^{p} e^{\alpha \frac{|x-y|^{2}}{t}} d x\right]^{1 / p} } & \leq \frac{C}{t^{1+k+n / 2 p^{\prime}}} \\
& \times[1+\sqrt{t} m(y, V)]^{-N} .
\end{aligned}
$$

(ii) For any given $p \in\left(1, p_{0}\right)$ with $p_{0}$ as in $(1.6), k \in \mathbb{Z}_{+}$and $N \in \mathbb{N}$, there exist positive constants $\alpha$ and $C$ such that, for any $t \in(0, \infty)$ and $y \in \mathbb{R}^{n}$,

$$
\left[\int_{\mathbb{R}^{n}}\left|[V(x)]^{1 / 2} \nabla_{x} \frac{\partial^{k}}{\partial t^{k}} K_{t}(x, y)\right|^{p} e^{\alpha \frac{|x-y|^{2}}{t}} d x\right]^{1 / p} \leq \frac{C}{t^{1+k+n / 2 p^{\prime}}}[1+\sqrt{t} m(y, V)]^{-N} .
$$

Remark 3.7. In particular, when $L=-\Delta+V$ is the Schrödinger operator, Lemma 3.6(i) is proved in [16, Proposition 3.3]. However, Lemma 3.6(ii) is new even in this case.

Proof of Lemma 3.6. We first prove (i) by considering two cases.

Case i): $k=0$. For any $t \in(0, \infty)$, choose a function $\phi^{t} \in C_{c}^{\infty}\left(\mathbb{R}^{n}\right)$ satisfying that

(i) $\operatorname{supp} \phi^{t} \subset B\left(\overrightarrow{0}_{n}, 2 \sqrt{t}\right), \phi^{t}(x) \equiv 1$ for any $x \in B\left(\overrightarrow{0}_{n}, \sqrt{t}\right),\left|\phi^{t}(x)\right| \leq 1$ for any $x \in \mathbb{R}^{n}$

(ii) there exists a positive constant $c$ such that, for any $x \in \mathbb{R}^{n},\left|\nabla \phi^{t}(x)\right| \leq$ $c / \sqrt{t},\left|\nabla^{2} \phi^{t}(x)\right| \leq c / t$.

For any $R \in(0, \infty)$ and $x \in \mathbb{R}^{n}$, let $\phi_{R}^{t}(x):=\phi^{t}(x / R)$. For any $t, R \in(0, \infty)$ and $x, y \in \mathbb{R}^{n}$, define

$$
w_{R}^{t}(x, y):=\phi_{R}^{t}(x-y) e^{\alpha \frac{|x-y|^{2}}{t}}
$$


where $\alpha$ is a positive constant which is determined later. Then, by a simple calculation, it is easy to see that $\operatorname{supp} w_{R}^{t}(\cdot, y) \subset B(y, 2 R \sqrt{t})$ and, for any $R \in$ $[1, \infty)$

$\left|w_{R}^{t}(x, y)\right| \leq e^{\alpha \frac{|x-y|^{2}}{t}},\left|\nabla_{x} w_{R}^{t}(x, y)\right| \leq \frac{c}{\sqrt{t}} e^{\alpha \frac{|x-y|^{2}}{t}}$ and $\left|\nabla_{x}^{2} w_{R}^{t}(x, y)\right| \leq \frac{c}{t} e^{\alpha \frac{|x-y|^{2}}{t}}$.

For any $t \in(0, \infty), R \in[1, \infty)$ and $y \in \mathbb{R}^{n}$, let

$$
\mathrm{I}_{R}^{t}(y):=\left\|w_{R}^{t}(\cdot, y) \nabla^{2} K_{t}(\cdot, y)\right\|_{L^{p}\left(\mathbb{R}^{n}\right)} .
$$

To estimate $\mathrm{I}_{R}^{t}(y)$, we make use of Lemma 3.3 with $f:=K_{t}(\cdot, y)$ and $\phi:=w_{R}^{t}(\cdot, y)$ therein. It is obvious that $w_{R}^{t}(\cdot, y) \in C_{c}^{\infty}\left(\mathbb{R}^{n}\right)$. Next we show that, for any $p \in(1, q], K_{t}(\cdot, y) \in W^{2, p}\left(\mathbb{R}^{n}\right)$. Indeed, by the fact $\nabla^{2} L^{-1}$ is bounded on $L^{p}\left(\mathbb{R}^{n}\right)$ for any $p \in(1, q], q \in(n / 2, \infty)$, (see Theorem $1.4($ iii) $)$ and $L\left(K_{t}(\cdot, y)\right)=$ $\frac{\partial}{\partial t} K_{t}(\cdot, y) \in L^{p}\left(\mathbb{R}^{n}\right)$, we find that

$$
\left\|\nabla^{2} K_{t}(\cdot, y)\right\|_{L^{p}\left(\mathbb{R}^{n}\right)}=\left\|\nabla^{2} L^{-1} L K_{t}(\cdot, y)\right\|_{L^{p}\left(\mathbb{R}^{n}\right)} \lesssim\left\|\frac{\partial}{\partial t} K_{t}(\cdot, y)\right\|_{L^{p}\left(\mathbb{R}^{n}\right)}<\infty .
$$

Thus, $K_{t}(\cdot, y) \in W^{2, p}\left(\mathbb{R}^{n}\right)$. From this and Lemma 3.3, we deduce that

$$
\begin{aligned}
\mathrm{I}_{R}^{t}(y) & \lesssim\left\|\nabla^{2} w_{R}^{t}(\cdot, y)\left|K_{t}(\cdot, y)\right|\right\|_{L^{p}\left(\mathbb{R}^{n}\right)}+\left\|\left|\nabla w_{R}^{t}(\cdot, y)\right| \mid \nabla K_{t}(\cdot, y)\right\| \|_{L^{p}\left(\mathbb{R}^{n}\right)} \\
& +\left\|w_{R}^{t}(\cdot, y) L_{0}\left(K_{t}(\cdot, y)\right)\right\|_{L^{p}\left(\mathbb{R}^{n}\right)} \\
= & : \mathrm{I}_{R, 1}^{t}(y)+\mathrm{I}_{R, 2}^{t}(y)+\mathrm{I}_{R, 3}^{t}(y) .
\end{aligned}
$$

For $\mathrm{I}_{R, 1}^{t}$, by (3.12) and (2.3), we conclude that, for any given $N \in \mathbb{N}$,

$$
\begin{aligned}
\mathrm{I}_{R, 1}^{t}(y) & =\left[\int_{\mathbb{R}^{n}}\left|\nabla_{x}^{2} w_{R}^{t}(x, y)\right|^{p}\left|K_{t}(x, y)\right|^{p} d x\right]^{1 / p} \\
& \lesssim \frac{1}{t^{1+n / 2}}[1+\sqrt{t} m(y, V)]^{-N}\left[\int_{\mathbb{R}^{n}} e^{(\alpha-c p) \frac{|x-y|^{2}}{t}} d x\right]^{1 / p} \\
& \lesssim \frac{1}{t^{1+n / 2 p^{\prime}}}[1+\sqrt{t} m(y, V)]^{-N} .
\end{aligned}
$$

To estimate $\mathrm{I}_{R, 2}^{t}$, by Lemma 3.5(i), we know that, for any given $N \in \mathbb{N}$, there exist positive constants $C, \alpha_{0}, c$ such that, for any $t \in(0, \infty)$ and $y \in \mathbb{R}^{n}$,

$$
\left[\int_{\mathbb{R}^{n}}\left|\nabla_{x} K_{t}(x, y)\right|^{p} e^{\alpha_{0} \frac{|x-y|^{2}}{t}} d x\right]^{\frac{1}{p}} \leq \frac{C}{t^{\frac{1}{2}+\frac{n}{2 p^{\prime}}}}[1+\sqrt{t} m(y, V)]^{-N} .
$$

From this and (3.12), we deduce that

$$
\begin{aligned}
\mathrm{I}_{R, 2}^{t}(y) & =\left[\int_{\mathbb{R}^{n}}\left|\nabla_{x} w_{R}^{t}(x, y)\right|^{p}\left|\nabla_{x} K_{t}(x, y)\right|^{p} d x\right]^{1 / p} \\
& \lesssim \frac{1}{t^{1 / 2}}\left[\int_{\mathbb{R}^{n}}\left|\nabla_{x} K_{t}(x, y)\right|^{p} e^{\alpha \frac{|x-y|^{2}}{t}} d x\right]^{1 / p} \\
& \lesssim \frac{1}{t^{1+n / 2 p^{\prime}}}[1+\sqrt{t} m(y, V)]^{-N},
\end{aligned}
$$


where the positive constant $\alpha$ as in (3.11) is chosen small enough such that $\alpha<\alpha_{0}$.

For $\mathrm{I}_{R, 3}^{t}$, by the fact that $L=L_{0}+V$ and $L\left(K_{t}(\cdot, y)\right)=\frac{\partial}{\partial t} K_{t}(\cdot, y)$, we find that

$$
\begin{aligned}
\mathrm{I}_{R, 3}^{t}(y) & =\left[\int_{\mathbb{R}^{n}}\left|w_{R}^{t}(x, y)\right|^{p}\left|(L-V)\left(K_{t}(\cdot, y)\right)(x)\right|^{2} d x\right]^{1 / p} \\
\leq & {\left[\int_{\mathbb{R}^{n}}\left|w_{R}^{t}(x, y)\right|^{p}\left|\frac{\partial}{\partial t} K_{t}(x, y)\right|^{p} d x\right]^{1 / p} } \\
& +\left[\int_{\mathbb{R}^{n}}\left|w_{R}^{t}(x, y)\right|^{p}\left|V(x) K_{t}(x, y)\right|^{p} d x\right]^{1 / p} \\
= & \mathrm{I}_{R, 3}^{t, 1}(y)+\mathrm{I}_{R, 3}^{t, 2}(y) .
\end{aligned}
$$

It follows from (3.12) and (2.7) that

$$
\begin{aligned}
\mathrm{I}_{R, 3}^{t, 1}(y) & \lesssim \frac{1}{t^{1+n / 2}}[1+\sqrt{t} m(y, V)]^{-N}\left[\int_{\mathbb{R}^{n}} e^{p(a-c) \frac{|x-y|^{2}}{t}} d x\right]^{1 / p} \\
& \lesssim \frac{1}{t^{1+n / 2 p^{\prime}}}[1+\sqrt{t} m(y, V)]^{-N},
\end{aligned}
$$

where the positive constant $c$ is as in (2.7) and $\alpha$ is chosen small enough such that $\alpha<c$.

For $\mathrm{I}_{R, 3}^{t, 2}$, by (3.12) and (2.3), we know that

$$
\begin{aligned}
\mathrm{I}_{R, 3}^{t, 2}(y) \lesssim & \frac{1}{t^{n / 2}}[1+\sqrt{t} m(y, V)]^{-N}\left\{\int_{\mathbb{R}^{n}}[V(x)]^{p} e^{p(\alpha-c) \frac{|x-y|^{2}}{t}} d x\right\}^{1 / p} \\
\lesssim & \frac{1}{t^{n / 2}}[1+\sqrt{t} m(y, V)]^{-N} \\
& \times \sum_{j=0}^{\infty}\left\{\int_{U_{j}(B(y, \sqrt{t}))}[V(x)]^{p} e^{-p c_{0} \frac{|x-y|^{2}}{t}} d x\right\}^{1 / 2},
\end{aligned}
$$

where $c_{0}:=(c-\alpha) \in(0, \infty)$ and $U_{j}(B(y, \sqrt{t}))$ is as in $(1.13)$. Since $V \in R H_{q}\left(\mathbb{R}^{n}\right)$ and $p \in(1, q]$, we know that $V \in R H_{p}\left(\mathbb{R}^{n}\right)$ and there exists some $p_{0} \in[1, \infty)$ such that $V \in A_{p_{0}}\left(\mathbb{R}^{n}\right)$. By this, we find that, for any $j \in \mathbb{Z}_{+}$,

$$
\begin{aligned}
& \left\{\int_{U_{j}(B(y, \sqrt{t}))}[V(x)]^{p} e^{-p c_{0} \frac{|x-y|^{2}}{t}} d x\right\}^{1 / p} \\
& \leq e^{-c_{0} 2^{2 j}}\left|B\left(y, 2^{j} \sqrt{t}\right)\right|^{\frac{1}{p}}\left\{\frac{1}{\left|B\left(y, 2^{j} \sqrt{t}\right)\right|} \int_{B\left(y, 2^{j} \sqrt{t}\right)}[V(x)]^{p} d x\right\}^{1 / p} \\
& \lesssim e^{-c_{0} 2^{2 j}}\left|B\left(y, 2^{j} \sqrt{t}\right)\right|^{-\frac{1}{p^{\prime}}} \int_{B\left(y, 2^{j} \sqrt{t}\right)}[V(x)] d x \\
& \lesssim e^{-c_{0} 2^{2 j}} 2^{-j n / p^{\prime}} 2^{j p 0 n} \frac{1}{t^{1-\frac{n}{2 p}}} \frac{1}{t^{\frac{n}{2}-1}} \int_{B(y, \sqrt{t})} V(x) d x,
\end{aligned}
$$

where, in the last inequality, we use the fact that $V \in A_{p_{0}}\left(\mathbb{R}^{n}\right)$ is a doubling measure (see, for example, [22, p. 196]), namely, there exists a positive constant 
$C$ such that, for any ball $B$ of $\mathbb{R}^{n}, V(2 B) \leq C 2^{p_{0} n} V(B)$. If $\sqrt{t} m(y, V)<1$, then, by Lemma 2.1(i), we know that

$$
\frac{1}{t^{\frac{n}{2}-1}} \int_{B(y, \sqrt{t})} V(x) d x \lesssim[\sqrt{t} m(y, V)]^{2-\frac{n}{q}} \lesssim 1 .
$$

If $\sqrt{t} m(y, V) \geq 1$, then, by Lemma 2.1(ii), we have

$$
\frac{1}{t^{\frac{n}{2}-1}} \int_{B(y, \sqrt{t})} V(x) d x \lesssim[\sqrt{t} m(y, V)]^{k_{0}},
$$

where $k_{0} \in(0, \infty)$ is as in Lemma 2.1(ii). From this, (3.17) and (3.18), it follows that

$$
\begin{aligned}
\mathrm{I}_{R, 3}^{t, 2}(y) \lesssim & \frac{1}{t^{n / 2}}[1+\sqrt{t} m(y, V)]^{-N} \frac{1}{t^{1-\frac{n}{2 p}}}\left\{1+[\sqrt{t} m(y, V)]^{k_{0}}\right\} \\
& \times \sum_{j=0}^{\infty} e^{-c_{0} 2^{2 j}} 2^{-j n / p} 2^{j p_{0} n} \\
& \lesssim \frac{1}{t^{1+n / 2 p^{\prime}}}[1+\sqrt{t} m(y, V)]^{-\left(N-k_{0}\right)} .
\end{aligned}
$$

This, combined with (3.16), implies that

$$
\mathrm{I}_{R, 3}^{t}(y) \lesssim \frac{1}{t^{1+n / 2 p^{\prime}}}[1+\sqrt{t} m(y, V)]^{-\left(N-k_{0}\right)} .
$$

By this, (3.14) and (3.13), we further conclude that

$$
\left\|w_{R}^{t}(\cdot, y) \nabla^{2} K_{t}(\cdot, y)\right\|_{L^{p}\left(\mathbb{R}^{n}\right)}=\mathrm{I}_{R}^{t}(y) \lesssim \frac{1}{t^{1+n / 2 p^{\prime}}}[1+\sqrt{t} m(y, V)]^{-\left(N-k_{0}\right)},
$$

where the implicit positive constant is independent of $R, t$ and $y$. Noticing that supp $\phi_{R}^{t}(\cdot / R) \subset B\left(\overrightarrow{0}_{n}, 2 R \sqrt{t}\right)$, via letting $R \rightarrow \infty$, we obtain

$$
\begin{aligned}
{\left[\int_{\mathbb{R}^{n}}\left|\nabla_{x}^{2} K_{t}(x, y)\right|^{p} e^{\alpha \frac{|x-y|^{2}}{t}} d x\right]^{1 / p} } & =\lim _{R \rightarrow \infty} \mathrm{I}_{R}^{t}(y) \\
& \lesssim \frac{1}{t^{1+n / 2 p^{\prime}}}[1+\sqrt{t} m(y, V)]^{-\left(N-k_{0}\right)} .
\end{aligned}
$$

For (3.10), we have

$$
\begin{aligned}
{\left[\int_{\mathbb{R}^{n}}\left|V(x) \frac{\partial^{k}}{\partial t^{k}} K_{t}(x, y)\right|^{p} e^{\alpha \frac{|x-y|^{2}}{t}} d x\right]^{1 / p} } & =\lim _{R \rightarrow \infty} \mathrm{I}_{R, 3}^{t, 2}(y) \\
& \lesssim \frac{1}{t^{1+n / 2 p^{\prime}}}[1+\sqrt{t} m(y, V)]^{-\left(N-k_{0}\right)}
\end{aligned}
$$

where $\mathrm{I}_{R, 3}^{t, 2}(y)$ is as in (3.15) and the last inequality follows from (3.19). Observing $N \in \mathbb{N}$ is arbitrary, we know that (3.9) and (3.10) hold true for any given $N \in \mathbb{N}$. This finishes the proof of (i) for $k=0$.

Case ii): $k \in \mathbb{N}$. In this case, by (2.7) and an argument similar to that used in the proof of [16, Proposition 3.3], we conclude that (3.9) and (3.10) hold true, which completes the proof of (i). 
Next, we prove (ii). If $q \in(n / 2, n)$, then $p \in\left(1, \frac{2 q n}{3 n-2 q}\right)$ and we could choose some positive constant $p_{1} \in\left(p, \frac{n q}{n-q}\right)$ such that $\frac{p p_{1}}{2\left(p_{1}-p\right)}<q$. If $q \in[n, \infty)$, then $p \in(1,2 q)$ and we could choose some positive constant $p_{1} \in(p, \infty)$ such that $\frac{p p_{1}}{2\left(p_{1}-p\right)}<q$. By this, the Hölder inequality and (3.8), we have

$$
\begin{aligned}
& \left\{\int_{\mathbb{R}^{n}}\left|[V(x)]^{1 / 2} \nabla_{x} \frac{\partial^{k}}{\partial t^{k}} K_{t}(x, y)\right|^{p} e^{\alpha \frac{|x-y|^{2}}{t}} d x\right\}^{1 / p} \\
& \leq\left\{\int_{\mathbb{R}^{n}}\left|\nabla_{x} \frac{\partial^{k}}{\partial t^{k}} K_{t}(x, y)\right|^{p_{1}} e^{\frac{2 \alpha p_{1}}{p} \frac{|x-y|^{2}}{t}} d x\right\}^{\frac{1}{p_{1}}} \\
& \quad \times\left\{\int_{\mathbb{R}^{n}}\left([V(x)]^{p / 2} e^{-\alpha \frac{|x-y|^{2}}{t}}\right)^{\left(p_{1} / p\right)^{\prime}} d x\right\}^{\frac{1}{p}-\frac{1}{p_{1}}} \\
& \left.\lesssim \frac{1}{t^{1 / 2+k+n / 2 p_{1}^{\prime}}}[1+\sqrt{t m}(y, V)]^{-N}[V(x)]^{\frac{p p_{1}}{2\left(p_{1}-p\right)}} d x\right\}^{\frac{1}{p}-\frac{1}{p_{1}}} \\
& \quad \times \sum_{j=0}^{\infty} e^{-c_{0} 2^{2 j}}\left\{\int_{U_{j}(B(y, \sqrt{t}))}\right. \\
& \lesssim \frac{1}{t^{1 / 2+k+n / 2 p_{1}^{\prime}}}[1+\sqrt{t} m(y, V)]^{-N} \sum_{j=0}^{\infty} e^{-c_{0} 2^{2 j}}\left|B\left(y, 2^{j} \sqrt{t}\right)\right|^{\frac{1}{p}-\frac{1}{p_{1}}} \\
& \quad \times\left\{\frac{1}{\left|B\left(y, 2^{j} \sqrt{t}\right)\right|} \int_{B\left(y, 2^{j} \sqrt{t}\right)}[V(x)]^{\frac{p p p_{1}}{2\left(p_{1}-p\right)}} d x\right\}^{\frac{1}{p}-\frac{1}{p_{1}}} .
\end{aligned}
$$

By the fact that $V \in R H_{q}\left(\mathbb{R}^{n}\right)$, we know that there exists some $p_{0} \in[1, \infty)$ such that $V \in A_{p_{0}}\left(\mathbb{R}^{n}\right)$. From this, the fact that $R H_{q}\left(\mathbb{R}^{n}\right) \subset R H_{\frac{p p_{1}}{2\left(p_{1}-p\right)}}\left(\mathbb{R}^{n}\right)$ and an argument similar to that used in (3.18), we deduce that

$$
\begin{aligned}
& \sum_{j=0}^{\infty} e^{-c_{0} 2^{2 j}}\left|B\left(y, 2^{j} \sqrt{t}\right)\right|^{\frac{1}{p}-\frac{1}{p_{1}}}\left\{\frac{1}{\left|B\left(y, 2^{j} \sqrt{t}\right)\right|} \int_{B\left(y, 2^{j} \sqrt{t}\right)}[V(x)]^{\frac{p p_{1}}{2\left(p_{1}-p\right)}} d x\right\}^{\frac{1}{p}-\frac{1}{p_{1}}} \\
& \lesssim \sum_{j=0}^{\infty} e^{-c_{0} 2^{2 j}}\left|B\left(y, 2^{j} \sqrt{t}\right)\right|^{\frac{1}{p}-\frac{1}{p_{1}}}\left\{\frac{1}{\left|B\left(y, 2^{j} \sqrt{t}\right)\right|} \int_{B\left(y, 2^{j} \sqrt{t}\right)} V(x) d x\right\}^{1 / 2} \\
& \lesssim \sum_{j=0}^{\infty} e^{-c_{0} 2^{2 j}} 2^{-j n\left(\frac{1}{2}-\frac{p_{1}-p}{p_{1} p}\right)} 2^{j n p_{0} / 2} t^{-\left[\frac{1}{2}-\frac{n\left(p_{1}-p\right)}{2 p_{1} p}\right]}\left[\frac{1}{t^{\frac{n}{2}-1}} \int_{B(y, \sqrt{t})} V(x) d x\right]^{1 / 2} \\
& \lesssim t^{-\left[\frac{1}{2}-\frac{n\left(p_{1}-p\right)}{2 p_{1} p}\right]} .
\end{aligned}
$$

This, combined with (3.20), finishes the proof of Lemma 3.6.

To prove Theorem 1.7, we also need the following atomic decomposition of $H_{L}^{p(\cdot)}\left(\mathbb{R}^{n}\right)$ (see (3.22) below), which is established in [26, Proposition 5.12].

Definition 3.8 ([26]). Let $p(\cdot) \in \mathcal{P}\left(\mathbb{R}^{n}\right)$ with $p_{+} \in(0,1]$ and $M \in \mathbb{N}$. A funtion

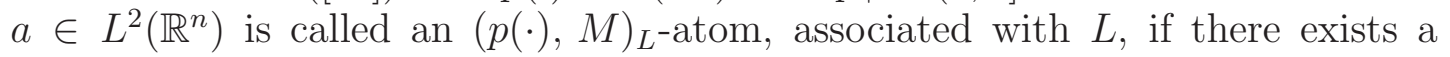


function $b \in \mathcal{D}\left(L^{M}\right)$ and a ball $B:=B\left(x_{B}, r_{B}\right)$ of $\mathbb{R}^{n}$ such that $a=L^{M}(b)$ and, for any $k \in\{0, \cdots, M\}$,

(i) $\operatorname{supp} L^{k}(b) \subset B$;

(ii) $\left\|\left(r_{B}^{2} L\right)^{k}(b)\right\|_{L^{2}\left(\mathbb{R}^{n}\right)} \leq r_{B}^{2 M}|B|^{1 / 2}\left\|\chi_{B}\right\|_{L^{p(\cdot)\left(\mathbb{R}^{n}\right)}}^{-1}$.

In what follows, for any $p(\cdot) \in \mathcal{P}\left(\mathbb{R}^{n}\right)$ with $0<p_{-} \leq p_{+} \leq 1$, any sequences $\left\{\lambda_{j}\right\}_{j \in \mathbb{N}} \subset \mathbb{C}$ and $\left\{B_{j}\right\}_{j \in \mathbb{N}}$ of balls in $\mathbb{R}^{n}$, define

$$
\mathcal{A}\left(\left\{\lambda_{j}\right\}_{j \in \mathbb{N}},\left\{B_{j}\right\}_{j \in \mathbb{N}}\right):=\left\|\left\{\sum_{j \in \mathbb{N}}\left[\frac{\left|\lambda_{j}\right| \chi_{B_{j}}}{\left\|\chi_{B_{j}}\right\|_{L^{p(\cdot)}\left(\mathbb{R}^{n}\right)}}\right]^{p_{-}}\right\}^{\frac{1}{p_{-}}}\right\|_{L^{p(\cdot)}\left(\mathbb{R}^{n}\right)} .
$$

Let $p(\cdot) \in C^{\log }\left(\mathbb{R}^{n}\right)$ with $p_{+} \in(0,1]$ and $M \in \mathbb{N} \cap\left(\frac{n}{2}\left[\frac{1}{p_{-}}-\frac{1}{2}\right], \infty\right)$. Then, from the fact that $L=-\operatorname{div}(A \nabla)+V$ is a nonnegative self-adjoint operator and $[26$, Proposition 5.12], we deduce that, for any $f \in H_{L}^{p(\cdot)}\left(\mathbb{R}^{n}\right) \cap L^{2}\left(\mathbb{R}^{n}\right)$, there exists a sequence $\left\{\lambda_{j}\right\}_{j \in \mathbb{N}} \subset \mathbb{C}$ and a family $\left\{a_{j}\right\}_{j \in \mathbb{N}}$ of $(p(\cdot), M)_{L}$-atoms, associated with balls $\left\{B_{j}\right\}_{j \in \mathbb{N}}$ of $\mathbb{R}^{n}$, such that

$$
f=\sum_{j=1}^{\infty} \lambda_{j} a_{j} \text { in } L^{2}\left(\mathbb{R}^{n}\right) \quad \text { and } \quad \mathcal{A}\left(\left\{\lambda_{j}\right\}_{j \in \mathbb{N}},\left\{B_{j}\right\}_{j \in \mathbb{N}}\right) \sim\|f\|_{H_{L}^{p(\cdot)}\left(\mathbb{R}^{n}\right)},
$$

where the implicit positive constants are independent of $f$.

The following lemma shows that the above atomic decomposition of $H_{L}^{p(\cdot)}\left(\mathbb{R}^{n}\right)$ allows one to reduce the study of the boundedness of operators on $H_{L}^{p(\cdot)}\left(\mathbb{R}^{n}\right)$ to studying their behaviours on single atoms.

Lemma 3.9. Let $L$ be as in $(1.1)$ and $p(\cdot) \in C^{\log }\left(\mathbb{R}^{n}\right)$ with $p_{+} \in(0,1]$. Suppose $T$ is a linear operator, or a positive sublinear operator, which is bounded on $L^{2}\left(\mathbb{R}^{n}\right)$. Let $M \in \mathbb{N} \cap\left(\frac{n}{2}\left[\frac{1}{p_{-}}-\frac{1}{2}\right], \infty\right)$. Assume that there exist positive constants $C$ and $\theta \in\left(n\left[\frac{1}{p_{-}}-\frac{1}{2}\right], \infty\right)$ such that, for any $(p(\cdot), M)_{L}$-atom a, associated with ball $B$ of $\mathbb{R}^{n}$, and $i \in \mathbb{Z}_{+}$,

$$
\|T(a)\|_{L^{2}\left(U_{i}(B)\right)} \leq C 2^{-i \theta}|B|^{\frac{1}{2}}\left\|\chi_{B}\right\|_{L^{p(\cdot)}\left(\mathbb{R}^{n}\right)}^{-1} .
$$

Then there exists a positive constant $C$ such that, for any $f \in H_{L}^{p(\cdot)}\left(\mathbb{R}^{n}\right)$,

$$
\|T(f)\|_{L^{p(\cdot)}\left(\mathbb{R}^{n}\right)} \leq C\|f\|_{H_{L}^{p(\cdot)}\left(\mathbb{R}^{n}\right)} .
$$

The proof of Lemma 3.9 is similar to that of [27, Corollary 3.16], the details being omitted.

We now prove Theorem 1.7.

Proof of Theorem 1.7. We first prove (1.9). By the fact that $q>\max \{n / 2,2\}$ and Theorem 1.4(i), we find that $V L^{-1}$ is bounded on $L^{2}\left(\mathbb{R}^{n}\right)$. By this and Lemma 3.9, to prove (1.9), it suffices to show that there exist positive constants $C$ and $\theta \in$ $\left(n\left[\frac{1}{p_{-}}-\frac{1}{2}\right], \infty\right)$ such that, for any $(p(\cdot), M)_{L^{-}}$atom $a$ with $M \in \mathbb{N} \cap\left(\frac{n}{2}\left[\frac{1}{p_{-}}-\frac{1}{2}\right], \infty\right)$, associated with ball $B:=B\left(x_{B}, r_{B}\right)$ of $\mathbb{R}^{n}$, and any $i \in \mathbb{Z}_{+}$,

$$
\left\|V L^{-1}(a)\right\|_{L^{2}\left(U_{i}(B)\right)} \leq C 2^{-i \theta}|B|^{\frac{1}{2}}\left\|\chi_{B}\right\|_{L^{p(\cdot)}\left(\mathbb{R}^{n}\right)}^{-1} .
$$


For any $i \in\{0, \ldots, 10\}$, since $V L^{-1}$ is bounded on $L^{2}\left(\mathbb{R}^{n}\right)$, we know that

$$
\left\|V L^{-1}(a)\right\|_{L^{2}\left(U_{i}(B)\right)} \lesssim\|a\|_{L^{2}(B)} \lesssim|B|^{1 / 2}\left\|\chi_{B}\right\|_{L^{p(\cdot)\left(\mathbb{R}^{n}\right)}}^{-1} .
$$

For any $i \in \mathbb{N}$ and $i \geq 11$, from the fact that $L^{-1}=\int_{0}^{\infty} e^{-t L} d t$, we deduce that

$$
\begin{aligned}
\left\|V L^{-1}(a)\right\|_{L^{2}\left(U_{i}(B)\right)} \leq & \left\|\int_{0}^{r_{B}^{2}} V(\cdot) e^{-t L}(a)(\cdot) d t\right\|_{L^{2}\left(U_{i}(B)\right)} \\
& +\left\|\int_{r_{B}^{2}}^{\infty} V(\cdot) e^{-t L}(a)(\cdot) d t\right\|_{L^{2}\left(U_{i}(B)\right)} \\
& =: \mathrm{I}_{i}+\mathrm{II}_{i} .
\end{aligned}
$$

We first estimate $\mathrm{I}_{i}$. By the Minkowski inequality, we find that

$$
\begin{aligned}
\mathrm{I}_{i} & =\left[\int_{U_{i}(B)}\left|\int_{0}^{r_{B}^{2}} V(x) e^{-t L}(a)(x) d t\right|^{2} d x\right]^{1 / 2} \\
& \leq \int_{0}^{r_{B}^{2}}\left[\int_{U_{i}(B)}\left|V(x) e^{-t L}(a)(x)\right|^{2} d x\right]^{1 / 2} d t .
\end{aligned}
$$

From the Minkowski inequality and the fact that supp $a \subset B$ (see Definition 3.8), we further deduce that,

$$
\begin{aligned}
& {\left[\int_{U_{i}(B)}\left|V(x) e^{-t L}(a)(x)\right|^{2} d x\right]^{1 / 2}} \\
& =\left[\int_{U_{i}(B)}\left|\int_{B} V(x) K_{t}(x, y) a(y) d y\right|^{2} d x\right]^{1 / 2} \\
& \leq \int_{B}|a(y)|\left[\int_{U_{i}(B)}\left|V(x) K_{t}(x, y)\right|^{2} d x\right]^{1 / 2} d y \\
& \leq \int_{B}|a(y)|\left[\int_{|x-y| \geq 2^{i-2} r_{B}}\left|V(x) K_{t}(x, y)\right|^{2} d x\right]^{1 / 2} d y .
\end{aligned}
$$

By applying Lemma 3.6(i) with $k=0$ and $p=2$ in (3.10), we obtain

$$
\begin{aligned}
& {\left[\int_{|x-y| \geq 2^{i-2} r_{B}}\left|V(x) K_{t}(x, y)\right|^{2} d x\right]^{1 / 2}} \\
& =\left[\int_{|x-y| \geq 2^{i-2} r_{B}}\left|V(x) K_{t}(x, y)\right|^{2} e^{\alpha \frac{|x-y|^{2}}{t}} e^{-\alpha \frac{|x-y|^{2}}{t}} d x\right]^{1 / 2} \\
& \leq e^{-\frac{\alpha}{8} \frac{2^{2 i} r_{B}^{2}}{t}}\left[\int_{|x-y| \geq 2^{i-2} r_{B}}\left|V(x) K_{t}(x, y)\right|^{2} e^{\alpha \frac{|x-y|^{2}}{t}} d x\right]^{1 / 2} \\
& \lesssim e^{-\frac{\alpha}{8} \frac{2^{2 i} r_{B}^{2}}{t}} \frac{1}{t^{1+n / 4}},
\end{aligned}
$$


where $\alpha \in(0, \infty)$ is as in (3.9). By this, (3.29), the Hölder inequality and Definition 3.8, we conclude that

$$
\begin{aligned}
& {\left[\int_{U_{i}(B)}\left|V(x) e^{-t L}(a)(x)\right|^{2} d x\right]^{1 / 2}} \\
& \quad \lesssim \int_{B}|a(y)| \frac{1}{t^{1+n / 4}} e^{-\frac{\alpha}{8} \frac{2^{2 i} r_{B}^{2}}{t}} d y \lesssim t^{-\left(1+\frac{n}{4}\right)} e^{-\frac{\alpha}{8} \frac{4^{i} r_{B}^{2}}{t}}\|a\|_{L^{2}(B)}|B|^{1 / 2} \\
& \quad \lesssim t^{-\left(1+\frac{n}{4}\right)} e^{-\frac{\alpha}{8} \frac{4^{i} r_{B}^{2}}{t}}|B|\left\|\chi_{B}\right\|_{L^{p(\cdot)}\left(\mathbb{R}^{n}\right)^{.}}^{-1}
\end{aligned}
$$

This, combined with (3.28), implies that

$$
\begin{aligned}
\mathrm{I}_{i} & \lesssim \int_{0}^{r_{B}^{2}} t^{-\left(1+\frac{n}{4}\right)} e^{-\frac{\alpha}{8} \frac{4^{i} r_{B}^{2}}{t}}\left\|\chi_{B}\right\|_{L^{p(\cdot)}\left(\mathbb{R}^{n}\right)}^{-1} d t \\
& \lesssim|B|\left\|\chi_{B}\right\|_{L^{p(\cdot)}\left(\mathbb{R}^{n}\right)}^{-1} \int_{0}^{r_{B}^{2}}\left(\frac{t}{4^{i} r_{B}^{2}}\right)^{N} t^{-\left(1+\frac{n}{4}\right)} d t \lesssim 2^{-2 i N}|B|^{1 / 2}\left\|\chi_{B}\right\|_{L^{p(\cdot)}\left(\mathbb{R}^{n}\right)}^{-1}
\end{aligned}
$$

where $N$ is a positive constant large enough such that $N>\frac{n}{4}$, which is determined later.

For $\mathrm{II}_{i}$, from the Minkowski inequality, we deduce that

$$
\begin{aligned}
\mathrm{II}_{i} & =\left[\int_{U_{i}(B)}\left|\int_{r_{B}^{2}}^{\infty} V(x) e^{-t L}(a)(x) d t\right|^{2} d x\right]^{1 / 2} \\
& \leq \int_{r_{B}^{2}}^{\infty}\left[\int_{U_{i}(B)}\left|V(x) e^{-t L}(a)(x)\right|^{2} d x\right]^{1 / 2} d t .
\end{aligned}
$$

Moreover, by (i) of Definition 3.8, we have

$$
e^{-t L}(a)=e^{-t L}\left(L^{M}(b)\right)=L^{M} e^{-t L}(b)=(-1)^{M} \frac{\partial^{M}}{\partial t^{M}} e^{-t L}(b) .
$$

By this, the Minkowski inequality, Lemma 3.6(i) and an argument similar to that used in (3.30), we know that

$$
\begin{aligned}
& {\left[\int_{U_{i}(B)}\left|V(x) e^{-t L}(a)(x)\right|^{2} d x\right]^{1 / 2}} \\
& =\left[\int_{U_{i}(B)}\left|\int_{B} V(x)\left(\frac{\partial^{M}}{\partial t^{M}} K_{t}(x, y)\right) b(y) d y\right|^{2} d x\right]^{1 / 2} \\
& \leq \int_{B}|b(y)|\left[\int_{|x-y| \geq 2^{i-2} r_{B}}\left|V(x)\left(\frac{\partial^{M}}{\partial t^{M}} p_{t}(x, y)\right)\right|^{2} d x\right]^{1 / 2} d y \\
& \lesssim \int_{B}|b(y)| t^{-\left(1+\frac{n}{4}+M\right)} e^{-c \frac{4^{i} r_{B}^{2}}{t}} d y \lesssim t^{-\left(1+\frac{n}{4}+M\right)} e^{-c \frac{4^{i} r_{B}^{2}}{t}}\|b\|_{L^{2}(B)}|B|^{1 / 2} \\
& \lesssim t^{-\left(1+\frac{n}{4}+M\right)} e^{-c \frac{4^{i} r_{B}^{2}}{t}} r_{B}^{2 M}|B|\left\|\chi_{B}\right\|_{L^{p(\cdot)\left(\mathbb{R}^{n}\right)}}^{-1}
\end{aligned}
$$


From this and (3.32), we deduce that

$$
\begin{aligned}
\mathrm{II}_{i} & \lesssim|B|\left\|\chi_{B}\right\|_{L^{p(\cdot)}\left(\mathbb{R}^{n}\right)}^{-1} 4^{-i M} \int_{r_{B}^{2}}^{\infty} t^{-\left(1+\frac{n}{4}\right)}\left(\frac{4^{i} r_{B}^{2}}{t}\right)^{M} e^{-c \frac{4^{i} r_{B}^{2}}{t}} d t \\
& \lesssim 2^{-2 i M}|B|^{1 / 2}\left\|\chi_{B}\right\|_{L^{p(\cdot)\left(\mathbb{R}^{n}\right)}}^{-1} .
\end{aligned}
$$

By this, (3.31) and (3.27), we find that

$$
\begin{aligned}
\left\|V L^{-1}(a)\right\|_{L^{2}\left(U_{i}(B)\right)} & \lesssim\left[2^{-2 i N}+2^{-2 i M}\right]|B|^{1 / 2}\left\|\chi_{B}\right\|_{L^{p(\cdot)}\left(\mathbb{R}^{n}\right)}^{-1} \\
& \lesssim 2^{-i \theta}|B|^{1 / 2}\left\|\chi_{B}\right\|_{L^{p(\cdot)}\left(\mathbb{R}^{n}\right)}^{-1}
\end{aligned}
$$

where $\theta:=\min \{2 N, 2 M\}$. By choosing $N>\frac{n}{2}\left(\frac{1}{p_{-}}-\frac{1}{2}\right)$ and the fact that $M \in$

$\mathbb{N} \cap\left(\frac{n}{2}\left[\frac{1}{p_{-}}-\frac{1}{2}\right], \infty\right)$, we find that $\theta \in\left(n\left[\frac{1}{p_{-}}-\frac{1}{2}\right], \infty\right)$. Hence, (3.25) holds true. This finishes the proof of (1.9).

By Lemma 3.6, the proofs of (1.10) and (1.11) are totally similar to that of (1.9), the details being omitted. Hence, we complete the proof of Theorem 1.7 .

Acknowledgments. Junqiang Zhang is very grateful to his advisor Professor Dachun Yang for his guidance and encouragements. Junqiang Zhang is supported by the Fundamental Research Funds for the Central Universities (Grant No. 2018QS01) and the National Natural Science Foundation of China (Grant No. 11801555). Zongguang Liu is supported by the National Natural Science Foundation of China (Grant No. 11671397).

\section{REFERENCES}

1. P. Auscher, X. T. Duong, and A. McIntosh, Boundedness of Banach space valued singular integral operators and Hardy spaces, unpublished manuscript, 2005.

2. P. Auscher and P. Tchamitchian, Square root problem for divergence operators and related topics, Astérisque 249 (1998), viii+172 pp.

3. M. Avellaneda and F. Lin, $L^{p}$ bounds on singular integrals in homogenization, Comm. Pure Appl. Math. 44 (1991), no. 8-9, 897-910.

4. J. Cao, D.-C. Chang, D. Yang, and S. Yang, Boundedness of second order Riesz transforms associated to Schrödinger operators on Musielak-Orlicz-Hardy spaces, Commun. Pure Appl. Anal. 13 (2014), no. 4, 1435-1463.

5. D. V. Cruz-Uribe and A. Fiorenza, Variable Lebesgue Spaces, Foundations and Harmonic Analysis, Applied and Numerical Harmonic Analysis, Birkhäuser/Springer, Heidelberg, 2013, x+312 pp.

6. D. Cruz-Uribe, A. Fiorenza, J. M. Martell, and C. Pérez, The boundedness of classical operators on variable $L^{p}$ spaces, Ann. Acad. Sci. Fenn. Math. 31 (2006), no. 1, 239-264.

7. D. Cruz-Uribe and L.-A. D. Wang, Variable Hardy spaces, Indiana Univ. Math. J. 63 (2014), no. 2, 447-493.

8. L. Diening, P. Harjulehto, P. Hästö, and M. Růžička, Lebesgue and Sobolev Spaces with Variable Exponents, Lecture Notes in Mathematics, 2017, Springer, Heidelberg, 2011.

9. X. T. Duong, S. Hofmann, D. Mitrea, M. Mitrea, and L. Yan, Hardy spaces and regularity for the inhomogeneous Dirichlet and Neumann problems, Rev. Mat. Iberoam. 29 (2013), no. 1, 183-236.

10. X. T. Duong and L. Yan, Duality of Hardy and BMO spaces associated with operators with heat kernel bounds, J. Amer. Math. Soc. 18 (2005), no. 4, 943-973. 
11. J. Dziubański and J. Zienkiewicz, $H^{p}$ spaces associated with Schrödinger operators with potentials from reverse Hölder classes, Colloq. Math. 98 (2003), no. 1, 5-38.

12. S. Hofmann, G. Lu, D. Mitrea, M. Mitrea, and L. Yan, Hardy Spaces Associated to Nonnegative Self-adjoint Operators Satisfying Davies-Gaffney Estimates, Mem. Amer. Math. Soc. 214 (2011), no. 1007, vi+78 pp.

13. S. Hofmann and S. Mayboroda, Hardy and BMO spaces associated to divergence form elliptic operators, Math. Ann. 344 (2009), no. 1, 37-116.

14. R. Jiang and D. Yang, Orlicz-Hardy spaces associated with operators satisfying DaviesGaffney estimates, Commun. Contemp. Math. 13 (2011), no. 2, 331-373.

15. K. Kurata and S. Sugano, A remark on estimates for uniformly elliptic operators on weighted $L^{p}$ spaces and Morrey spaces, Math. Nachr. 209 (2000), 137-150.

16. F. K. Ly, Second order Riesz transforms associated to the Schrödinger operator for $p \leq 1$, J. Math. Anal. Appl. 410 (2014), no. 1, 391-402.

17. F. K. Ly, Classes of weights and second order Riesz transforms associated to Schrödinger operators, J. Math. Soc. Japan 68 (2016), no. 2, 489-533.

18. E. Nakai and Y. Sawano, Hardy spaces with variable exponents and generalized Campanato spaces, J. Funct. Anal. 262 (2012), no. 9, 3665-3748.

19. W. Orlicz, Über konjugierte Exponentenfolgen, Studia Math. 3 (1931), 200-211.

20. Y. Sawano, Atomic decompositions of Hardy spaces with variable exponents and its application to bounded linear operators, Integral Equations Operator Theory 77 (2013), no. 1, 123-148.

21. Z. Shen, $L^{p}$ estimates for Schrödinger operators with certain potentials, Ann. Inst. Fourier (Grenoble) 45 (1995), no. 2, 513-546.

22. E. M. Stein, Harmonic Analysis: Real-Variable Methods, Orthogonality, and Oscillatory Integrals, Princeton Mathematical Series, 43, Monographs in Harmonic Analysis, III, Princeton University Press, Princeton, NJ, 1993, xiv+695 pp.

23. L. Tang, Weighted norm inequalities for Schrödinger type operators, Forum Math. 27 (2015), no. 4, 2491-2532.

24. L. Yan, Classes of Hardy spaces associated with operators, duality theorem and applications, Trans. Amer. Math. Soc. 360 (2008), no. 8, 4383-4408.

25. S. Yang, Some estimates for Schrödinger type operators on Musielak-Orlicz-Hardy spaces, Taiwanese J. Math. 18 (2014), no. 4, 1293-1328.

26. D. Yang and J. Zhang, Variable Hardy spaces associated with operators satisfying DaviesGaffney estimates on metric measure spaces of homogeneous type, Ann. Acad. Sci. Fenn. Math. 43 (2018), no. 1, 47-87.

27. D. Yang, J. Zhang, and C. Zhuo, Variable Hardy spaces associated with operators satisfying Davies-Gaffney estimates, Proc. Edinb. Math. Soc. 61 (2018), no. 3, 759-810.

28. D. Yang and C. Zhuo, Molecular characterizations and dualities of variable exponent Hardy spaces associated with operators, Ann. Acad. Sci. Fenn. Math. 41 (2016), no. 1, 357-398

29. J. Zhong, Harmonic Analysis for Some Schrödinger Type Operators, Ph.D. Thesis, Princeton University, 1993.

${ }^{1}$ School of Science, China University of Mining and Technology-Beijing, BeiJing 100083, PeOple's Republic of China

E-mail address: jqzhang@cumtb.edu.cn; zhangjunqiang@mail.bnu.edu.cn

${ }^{2}$ School of Science, China University of Mining and Technology-Beijing, BeiJing 100083, People's Republic of China

E-mail address: liuzg@cumtb.edu.cn 